“(C) 2014 IEEE. Personal use of this material is permitted. Permission from IEEE must be obtained for all other uses, in any current or future media, including reprinting/republishing this material for advertising or promotional purposes, creating new collective works, for resale or redistribution to servers or lists, or reuse of any copyrighted component of this work in other works." 


\title{
Detection of Denial-of-Service Attacks Based on Computer Vision Techniques
}

\author{
Zhiyuan Tan, Member, IEEE, Aruna Jamdagni, Xiangjian $\mathrm{He}^{\ddagger}$, Senior Member, IEEE, \\ Priyadarsi Nanda, Senior Member, IEEE, Ren Ping Liu, Senior Member, IEEE, and Jiankun Hu, Member, IEEE
}

\begin{abstract}
Detection of Denial-of-Service (DoS) attacks has attracted researchers since 1990s. A variety of detection systems has been proposed to achieve this task. Unlike the existing approaches based on machine learning and statistical analysis, the proposed system treats traffic records as images and detection of DoS attacks as a computer vision problem. A multivariate correlation analysis approach is introduced to accurately depict network traffic records and to convert the records into the respective images. The images of network traffic records are used as the observed objects of our proposed DoS attack detection system, which is developed based on a widely used dissimilarity measure, namely Earth Mover's Distance (EMD). EMD takes cross-bin matching into account and provides a more accurate evaluation on the dissimilarity between distributions than some other well-known dissimilarity measures, such as Minkowskiform distance $L_{p}$ and $X^{2}$ statistics. These unique merits facilitate our proposed system with effective detection capabilities. To evaluate the proposed EMD-based detection system, ten-fold cross-validations are conducted using KDD Cup 99 data set and ISCX 2012 IDS Evaluation data set. The results presented in the system evaluation section illustrate that our detection system can detect unknown DoS attacks and achieves $99.95 \%$ detection accuracy on KDD Cup 99 data set and $90.12 \%$ detection accuracy on ISCX 2012 IDS evaluation data set with processing capability of approximately 59,000 traffic records per second.
\end{abstract}

Index Terms-Denial-of-Service, anomaly-based detection, earth mover's distance, computer vision

\section{INTRODUCTION}

D ENIAL-OF-SERVICE (DoS) attacks have emerged as one of the most severe network intrusive behaviours and have posed serious threats to the infrastructures of computer networks and various network-based services [1]. These attacks can be launched by deliberately exploiting system vulnerabilities of a victim (e.g., a host, a router, or an entire network) or flooding a victim with a large volume of useless network traffic to occupy the designated resources (e.g., network bandwidth, processor time and memory). DoS attacks can result in a serious interruption to a victim. Moreover, in

Z. Tan is with the Services, Cybersecurity and Safety Group, University of Twente, Enschede, Netherlands. E-mail: Z.Tan@utwente.nl.

$X$. He, and P. Nanda are with the Centre for Innovation in IT Services and Applications (iNEXT), University of Technology, Sydney, Australia. E-mail: Xiangjian.He, Priyadarsi.Nanda@uts.edu.au.

A. Jamdagni is with School of Computing and Mathematics, University of Western Sydney, Parramatta, Australia. E-mail: a.jamdagni@uws.edu.au.

R. Liu is with the Information and Communication Technologies (ICT) Centre, Commonwealth Scientific and Industrial Research Organisation (CSIRO), Marsfield, Australia. E-mail: ren.liu@csiro.au.

J. Hu is the Professor of Cyber Security at the Canberra Campus of the University of New South Wales. E-mail: J.Hu@adfa.edu.au

$\ddagger$ Corresponding author: X. He. today's Internet, attack toolkits are readily available and easy to use [2] [3]. Any Internet users can use these toolkits to launch attacks with minimum efforts. Sometimes, the users of the attack toolkits may not even have any knowledge about network security.

Therefore, a significant number of works in recent years have concentrated on building systems for defending DoS attacks. The defence mechanisms residing in these systems are generally classified as detection, prevention, mitigation and response [4]. Detection is the very first step to protect against DoS attacks among the aforementioned defence mechanisms, and it is required to provide prompt reaction and high detection accuracy.

In general, detection mechanisms can be divided into two major categories, namely misuse-based detection and anomaly-based detection. The former detection mechanism employs signature or rule matching in its recognition of intrusive behaviours. Systems based on misuse detection mechanism can achieve high detection rates in known attacks [5]-[7]. However, they are incapable of detecting any unknown malicious behaviours or even variants of existing attacks. Furthermore, generating signatures for previously unseen attacks is a labour intensive task, which heavily involves network security expertise. In contrast, anomaly-based detection mechanism uses a different detection methodology that monitors and labels any network activities presenting significant deviation from the respective legitimate traffic profiles as suspicious objects. Since these profiles are built on the knowledge of normal network behaviours, anomaly-based detection mechanism is able to identify previously unknown attacks. As such, it is widely adopted in the research community [8].

Since the last decade, a variety of anomaly-based detection systems has been proposed. However, the existing systems suffer from a common issue in achieving high accuracy in classifying both normal traffic and attack traffic [9]. This is partly because most of these systems only use several simple network features of incoming traffic (e.g., IP header fields) in modelling normal network traffic, and overlook the correlations between the network features. Though there is a current research trend to make use of the correlations between the features in intrusion detection, most of the proposed systems [10]-[12] are based on traditional statistical correlation analysis techniques, which are only capable of studying the correlations between the features (variables) in a given sample set. The properties inherited from these traditional statistical correlation analysis techniques make these anomaly-based detection systems incapable of recognising individual attack 
records hidden in a sample set.

In addition, more sophisticated classifiers are demanded to help improve detection accuracy. The techniques used in computer vision tasks are the potential candidates. Due to some commonalities shared between DoS attack detection and computer vision tasks, such as image retrieval and object shape recognition. Normal traffic to DoS attack detection can be equivalent to queries to image retrieval tasks or object shape recognition tasks. DoS attacks to our detection task can be interpreted as the images or the object shapes that do not match the queries. Therefore, computer vision techniques can provide intuitive and effective solutions to the problem.

In this paper, we propose a more sophisticated anomalybased system for detecting DoS attacks. The proposed system is designed to overcome all the aforementioned issues and to solve the detection problem from the perspective of computer vision. Our system has three key features:

- First, the hidden correlations between the features of network traffic are extracted using our previously developed Multivariate Correlation Analysis (MCA) technique [13], which provides accurate network traffic characterisation,

- Second, individual attack records hidden in the crowd can be easily recognised by our system. This is owing to one of the merits (i.e., the capability of analysing correlation between features within individual records) of our MCA technique.

- Finally, to improve the detection accuracy, our proposed system adopts the principle of object shape recognition and Earth Mover's Distance (EMD) [14] (a robust distance metric) in the design of attack detectors. To the best of our knowledge, it is the first time that EMD has ever been applied to the field of network DoS attack detection.

This new anomaly-based DoS attack detection system differs the work presented in this paper from our recent study published in [13]. To improve the accuracy and to accelerate the computation of our MCA approach [13], Principal Component Analysis (PCA) is employed in this new detection system to reduce the dimensionality (noise) of data. Furthermore, unlike the previous work, inbound network traffic records are converted into two-dimensional images before detection is conducted. More importantly, EMD instead of Mahalanobis distance is utilised in this work to measure the dissimilarity between observed inbound traffic records and a pre-built normal profile.

The proposed DoS attack detection system is evaluated using the KDD Cup 99 data set [15] and ISCX 2012 IDS evaluation data set [16] on DoS attacks. The experimental results on these two data sets are compared against three stateof-the-art detection systems (i.e., network intrusion detection system based on covariance feature space [11], triangle-areabased nearest neighbours approach [12] and DoS attack detection system using TAM-based MCA [13]) and four Naive Bayes (NB) based detection approaches [46] respectively. The overall evaluation shows that our detection system achieves 99.95\% accuracy on KDD Cup 99 data set, which outperforms the systems discussed in [11] and [12] by $2.06 \%$ and $7.8 \%$ respectively and is as good as the system suggested by [13]. Meanwhile, our proposed detection system achieves $90.12 \%$ accuracy on the up-to-date ISCX 2012 IDS evaluation data set, which shows advantages over the four NB-based detection approaches [46]. The computational complexity of our system is then discussed and compared with the two state-of-theart detection systems, which also employ correlation analysis techniques in design.

The rest of this paper is organised as follows. We present a review on prior research works on anomaly-based detection and EMD in Section II. Section III proposes a new DoS attack detection system based on computer vision techniques. Section IV illustrates performance evaluations of our proposed detection system on KDD Cup 99 data set and ISCX 2012 IDS evaluation data set. Section V presents a systematic analysis on the computational complexity and the time cost of the proposed detection system. Finally, conclusions are drawn in Section VI.

\section{RELATED WORKS}

In order to provide more detailed background information about our work, a literature review is conducted in this section. However, our intention is not to give a comprehensive survey on the topic. Instead, we only cover the most related studies on anomaly-based detection, EMD and the applications of EMD in the field of network security. Moreover, as our work employs the mechanism of network-based anomaly intrusion detection, all the detection systems covered in this section are limited to network-based systems unless and otherwise being specified.

\section{A. Anomaly-based Detection}

Anomaly-based detection mechanism shows promising results in detecting zero-day attacks [17] that exploit previously unknown system vulnerability, and it has less dependency on domain knowledge. Recent work on DoS attack detection primarily adopts this concept. Techniques used in these anomalybased detection systems can be divided into two categories, namely machine learning and statistical analysis.

Machine learning techniques help in classification of observed objects using known properties learnt from training data. Lee et al. [18] built a Distributed Denial-of-Service (DDoS) attack detection approach based on hierarchical clustering method. The approach could detect different phases of a DDoS attack instance. However, the final detection accuracy of the approach was not revealed. Tajbakhsh et al. [19] proposed two classification approaches, called Association Based Classification (ABC) and ABC extension. Models of different classes were described using fuzzy association rules. The ABC and the $\mathrm{ABC}$ extension were applied for misuse-based detection and anomaly-based detection respectively. They achieved encouraging results except on novel attacks. Mukkamala et al. [20] proposed an ensemble design of intrusion detection system, where Artificial Neural Networks (ANN), Support Vector Machines (SVM) and Multivariate Adaptive Regression Splines (MARS) techniques were used. The experimental results show that this system achieves $99.97 \%$ detection accuracy and outperforms any of the individual techniques. However, 
the ensemble detection system involves time-consuming computation and cannot work real-time. Yu et al. [21] suggested a two-tier hierarchical detection system using SVM. The hierarchical structure and one-class SVM (i.e., Support Vector Data Description) equip it with the advantage in classifying various attacks into their appropriate classes. This detection system achieved its best attack detection rate of $99.40 \%$ using 3 selected Management Information Based (MIB) features.

Statistical analysis techniques have been employed to conduct investigation into attributes of network traffic packets and to determine a rationale threshold for discriminating attacks from the legitimate traffic. Wang et al. [22] proposed a sequential Change-Point Monitoring (CPM) approach for the detection of DoS attacks. A non-parametric Cumulative Sum (CUSUM) algorithm was used in the CPM to evaluate the significance of the changes of traffic patterns and to determine the appearance of DoS attacks. The CPM is more suitable for analysing a complex network environment. Whereas in [22], CPM was only tested using SYN flooding attacks. Moreover, its performance is possibly affected by network indiscipline. Kim and Reddy [23] suggested a statistical-based approach to detect anomalies at an egress router. Discrete wavelet transform was used to transform address correlation data (i.e., the correlation of destination IP addresses, port numbers and the number of flows). This statistical-based detection technique provides a solution to detect outgoing anomalous traffic at source networks. Thatte et al. [24] developed a bivariate Parametric Detection Mechanism (bPDM) operating on aggregate traffic. The bPDM applies the Sequential Probability Ratio Test (SPRT) on two aggregate traffic statistics (i.e., packet rate and packet size), and it alleges an anomaly only when a rise in the traffic volume is associated with a change in the distribution of packet-size.

Despite the afore-discussed systems or approaches show innovation and promise in different aspects of attack detection, they still suffer from relatively high false positive rates. This is partly because they either neglect the dependency and correlation between features/attributes or do not manage to fully exploit the correlation [25]. Some recent studies attempt to cope with this problem by taking full advantage of the correlation in their designs. Thottan and Ji [10] developed an abrupt change detection approach which employs statistical signal processing technique based on the Auto-Regression (AR) process. An operation matrix $(A)$, which retained "the ensemble average of the two point spatial cross-correlation of the abnormality vectors estimated over a time interval $T$ " [10], participated in the computation of the value of abnormality indicator. Although this detection approach has shown to be effective in detecting several network anomalies, it is still an open topic for now how to manage features with various time granularities. Jin et al. [11] proposed a statistical detection approach using covariance matrix to represent the multivariate correlation for sequential samples. Although the approach achieves good detection rates, it is vulnerable to attacks that linearly change all monitored features. Moreover, it can only label a group of observed samples as legitimate or attack traffic without distinguishing individual attack traffic records from the crowd. Tsai and Lin [12] designed a new detection approach based on the nearest neighbours technique. The approach applied a triangle area based method to discover the correlation between observed objects and the cluster centroids pre-identified using the $K$-means algorithm. The extracted correlation was then used in the nearest neighbours algorithm for classification. Though this detection approach was carefully designed to be immune to the problem of linear changing features, the dependency on prior knowledge of anomalous behaviours dilutes its accuracy and reliability on correlation discovery. The detection effectiveness of these systems is reported in Section IV-C.

In our previous works [13] [26], mechanisms to overcome the above weaknesses were studied and the corresponding solutions were proposed. A multi-tier Real-time Payloadbased IDS (RePIDS) was proposed in [26], where a novel geometrical structure based analysis technique was deliberately designed for feature correlation extraction. Mahalanobis Distance Map (MDM) was used to reveal the correlation between packet payload features. In [13], we attempted to remove the dependency on network traffic packet payload by diverting to connection-based features. This eliminates the restriction of the use of IDS to encrypted network traffic. A Multivariate Correlation Analysis (MCA) approach proposed in [13] embraces triangle area in estimating the correlation between features. This MCA approach equips our proposed DoS attack detection system with encouraging detection accuracy and higher efficiency. The details of the MCA approach will be discussed in Section III-A5a. However, the previously proposed MCA-based detection system is based on Mahalanobis distance, which does not support partial matching. A more sophisticated distance metric, such as the EMD, can enhance the accuracy of detection. Detailed introduction and discussion will be presented in Sections II-B and III-A4.

In addition, although the work shown in [27] [28] [29] demonstrates good attempts of adopting some ideas of computer vision into intrusion detection problems, these schemes do not take into account the correlation between various features. Specifically, they are only proposed to represent instances of network packet header data (e.g., traffic volume or port numbers) as images. In comparison, however, to reformulate the intrusion detection problem as a computer vision task can further exploit the merits of this innovative fusion and motivate research on this topic.

\section{B. Earth Mover's Distance}

Earth Mover's Distance (EMD) was originally proposed by Rubner et al. [30] as a cross-bin dissimilarity measure to evaluate the perceptual difference between two distributions. It was defined as the minimal cost of the transformation from one distribution to another. EMD supports partial matching and outperforms bin-by-bin distances in matching perceptual dissimilarity. This benefits from the extension of the concept of a distance from between corresponding elements to between the entire distributions, in which the ground distance reflects the notion of nearness between the elements in the distributions. Quantisation and other binning problems of histograms can be further avoided by taking the above ideas. Further 
discussion on the theoretical advantages and suitability of computer vision techniques, including EMD, in DoS detection can be found in Section III-A4

1) Earth Mover's Distance Approaches: A considerable amount of research interest on EMD has been raised by the early work [30] [31] from Rubner et al., who adopted transportation problem [32] in modelling distribution comparison and suggested to compare the signatures of distributions rather than to compare histograms. The computation time of EMD is reduced owing to the advantage that signatures are usually the compressed (clustered) versions of histograms. However, simplex algorithm [33], applied to solve EMD, has a supercubic empirical time complexity in $\Omega\left(N^{3}\right) \cap O\left(N^{4}\right)$ for a signature with $N$ elements, which limits the applications of EMD to non-time-sensitive tasks mostly. Grauman and Darrell [34] proposed a fast contour matching algorithm using an approximate EMD, which utilised embedding technique to accelerate the computational speed. Thus, the EMD between two sets of descriptive local features can be quickly computed in the complexity of $O(N d \log (\triangle))$, where $N$ is the number of features, $d$ is their dimension, and $\triangle$ is the diameter of the feature space. Moreover, Ling and Okada [14] suggested an alternative fast version for EMD in which $L_{1}$ distance was used as ground distance to compute the dissimilarity between histograms. An efficient tree-based algorithm was developed replacing the original simplex algorithm to solve the proposed EMD- $L_{1}$ in a more efficient fashion. It was shown in [14] that EMD- $L_{1}$ had an average empirical complexity of $O\left(N^{2}\right)$ that was computationally much less expensive than the original EMD. EMD- $L_{1}$ was applied to shape recognition and interest point matching. Based on the same motivation that was to speed up the original EMD, Differential Earth Mover's Distance (DEMD) was recently presented in [35]. The authors proposed applying sensitivity analysis of the simplex algorithm to solve EMD. The signatures of distributions were used to represent the interested objects in visual tracking. Considering the efficiency and the scenarios for which the above approaches were proposed, EMD $L_{1}$ is believed to be the best candidate for our task.

2) Applications of Earth Mover's Distance in Network Security: EMD has been widely used to solve many problems in computer vision, such as image retrieval [30] [31], contour matching [34], object shape recognition [14], interest point matching [14] and visual tracking [35] etc. It is still a new technique to computer and network security, and only a small amount of work based on EMD has been found in the literature.

In this paragraph, some of the most closely related works on intrusive behaviour detection are introduced. For instance, an approach for phishing web page detection was presented in [36], where web pages were first converted into normalised images and then were described using signatures (i.e., features consisting of dominant colour category and the respective centroid coordinates). Visual similarities between a test web page and protected web pages were assessed using the EMD [31] between their image signatures. If the similarity between the tested web page and a particular protected web page exceeds the pre-defined threshold, the tested page is deemed as a phishing web page. In [37], Yen and Reiter developed a test method to differentiate between Plotters (i.e., bots) and Traders (i.e., normal peers) on a Peer-to-Peer (P2P) network. EMD [31] helped evaluate the similarity between the per-destination interstitial time distributions of hosts. Plotters normally showed similar patterns in distribution, but those of Traders tended to be far apart from each other. The hosts were then grouped into the clusters with respect to the similarity of their timing patterns. Micarelli and Sansonetti proposed a case-based anomaly intrusion detection approach in [39]. This approach monitored the output parameters and the arguments of system calls (i.e., execve(), $\operatorname{chmod}(), \operatorname{chown}()$, exit(), open() and setuid()) revoked by instances of applications on a host. A signature (consisting of the centroids of the clusters of system calls and the corresponding weights) was used to represent an instance of an application. Then, the signature was compared with the case (represented by the signature of the generic instance of the same application) stored in the profile database using EMD [31]. Behaviours of the system call sequences performing significantly non-compliant with the corresponding profiles inferred that attacks were underway.

Although the above studies have made contributions to the integration between EMD and the respective proposed detection approaches, none of the approaches has been designed particularly for DoS attack detection. Additionally, these studies employ the original EMD rather than any other enhanced versions. The heavy computational complexity of the original EMD prevents them from being applied in prompt detection tasks. The theoretical advantages of EMD and the shortcomings in recent applications of EMD motivate us to explore a better means to integrate EMD- $L_{1}$ (a fast version of EMD) and DoS attack detection task.

\section{DoS ATtaCK Detection System}

As a core component of a comprehensive network security scheme, a DoS attack detection system defends internal networks under the same administrative control from being affected by the imposed malicious traffic. An overview of our proposed DoS attack detection system architecture is given in this section, in which detection mechanisms, system framework and relevant algorithms are discussed. In particular, the advantages and suitability of using computer vision techniques in DoS attack detection are discussed in Section III-A4.

\section{A. General Mechanisms of the Detectors}

1) Traffic Monitoring at the Destination: Our proposed DoS attack detection system is deployed at the gateway of a network to monitor and analyse incoming network traffic. This reduces the overhead in detecting malicious activities by concentrating only on relevant inbound traffic. This also enables our detector to provide protection which is best fit for the targeted internal network because legitimate traffic profiles residing in the detectors are developed for a smaller number of network services.

2) Sample-by-sample Detection: Our system investigates traffic samples individually in the process of detection. This releases our system from the dependency on the assumption 
made by the group-based detection mechanism [11] that the network traffic samples in a tested group are all from the same distribution (class). Moreover, our approach can detect attacks in a prompt manner with less delay than the groupbased detection approach. Besides, it has been proven that the sample-by-sample detection mechanism can always achieve equal or better detection precision than the group-based detection mechanism in a general network scenario [13].

3) Anomaly-based Detectors: Anomaly-based detection mechanism [8] is adopted in our approach. It facilitates the detection of any DoS attacks demonstrating deviation from the normal traffic profiles without requiring any relevant expertise. Thus, labour-intensive attack analysis and frequent update of attack signature database in the case of misuse-based detection system are avoided. Meanwhile, the mechanism enhances the robustness of the proposed detectors and makes them harder to be evaded since the dependency on attack signatures has been disconnected. Moreover, without knowing the exact detection algorithm in use, attackers can merely get the way to penetrate the defence. This is because they need to generate an attack that can match the legitimate traffic profiles built by the specific detection algorithm, which however is a labourintensive work, and in-depth expertise in the targeted detection algorithm is required.

4) Attack Recognition Based on Computer Vision: The commonalities shared between a DoS attack detection problem and computer vision tasks (e.g., image retrieval and object shape recognition) encourage us adopt the principals used in computer vision into the task of this paper. Normal traffic profiles to our DoS attack detection system are treated as queries to image retrieval tasks or shape recognition tasks. Instances of normal traffic, on one hand, are interpreted as the images or the shapes that match the queries. DoS attacks, on the other hand, are interpreted as the unmatched images or the unmatched shapes. The ideas and techniques used in computer vision tasks can be introduced to solve the problems of DoS attack detection. Moreover, computer vision techniques, namely EMD and its variants, make use of crossbin correlation in assessing perceptional dissimilarity between two images, which contributes higher accuracy than other binto-bin dissimilarity measures (e.g., $L_{1}, L_{2}$ and $X^{2}$ distances) [14]. This coincides to one of the aims of our work that exploiting correlation of features in detection. In addition, partial matching, another merit supported by EMD and its variants, helps further enhance the detection accuracy of the proposed detection system. This is because this merit allows our system to adjust its degree of tolerance to the variance of normal network traffic.

a) EMD- $L_{1}$ : Earth Mover's Distance (EMD) [31] was originally inspired by the intuition that looking for a solution with the minimum overhead on moving a mass of earth properly spreading in space to a collection of holes in the same space. Despite working effectively, the expensive computation restricts the applications of EMD mainly in offline tasks. Subsequent research on EMD suggests various techniques to alleviate the overhead of computation. An equivalent simplification, EMD- $L_{1}$ [14], introduces a new efficient formulation of the EMD between histograms (a special type of signatures with

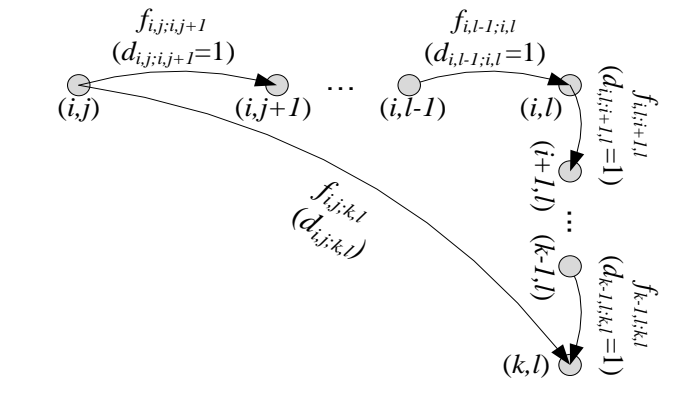

Remark: $d_{i, j ; k, l}=d_{i, j ; i, j+l}+\ldots+d_{i, l-l ; i, l}+d_{i, l ; i+1, l}+\ldots+d_{k-1, l ; k, l}$

Fig. 1. Decompose a flow

non-sparse structures). $L_{1}$ (i.e., Manhattan) distance is chosen as the ground distance in this new formulation, which redefines the computation of EMD as a "network flow problem".

With the new formulation, the computational complexity of EMD can be reduced by one order of magnitude in comparison with the original formulation using transportation problem. This is owing to an important property of the $L_{1}$ distance that any shortest path between two points on a network can be decomposed into a collection of edges between neighbour nodes with a ground distance of one between them. As shown in Fig. 1, the shortest path between the node $(i, j)$ and the node $(k, l)$, where $i<k$ and $j<l$, is decomposed into a collection of edges (including $f_{i, j ; i, j+1}, f_{i, l-1 ; i, l}, f_{i, l ; i+1, l}$, $f_{k-1, l ; k, l}$ etc.) with ground distances of ones, and its distance is defined as the summation of the distances of the edges (i.e., $\left.d_{i, j ; k, l}=d_{i, j ; i, j+1}+\cdots+d_{i, l-1 ; i, l}+d_{i, l ; i+1, l}+\cdots+d_{k-1, l ; k, l}\right)$.

Using the following notations, the new formulation of EMD (i.e., EMD- $L_{1}$ ) considering only the flows (edges) between neighbour bins (nodes) is defined. Without loss of generality, we assume there are two-dimensional histograms with $k$ rows and $q$ columns and $N=k \times q$ bins. $\mathcal{I}=\{(j, p): 1 \leq j \leq$ $k, 1 \leq p \leq q\}$ is an index set where $(j, p)$ indicates the index of a bin (or node) within a histogram. $\mathcal{J}=\{(j, p, c, d)$ : $(j, p) \in \mathcal{I},(c, d) \in \mathcal{I}\}$ is an index set where $(j, p, c, d)$ is the index of a flow $f_{j, p ; c, d}$ from bin $(j, p)$ to bin $(c, d)$. $\mathcal{J}_{1}=$ $\left\{(j, p, c, d):(j, p, c, d) \in \mathcal{J}, d_{j, p ; c, d}=1\right\}$ denotes an index set where $(j, p, c, d)$ is the index of a flow $f_{j, p ; c, d}$ from bin $(j, p)$ to bin $(c, d)$, and the two bins are neighbours with a ground distance of one.

Given histogram $Y=\left\{y_{j p}:(j, p) \in \mathcal{I}\right\}$, where $y_{j p}$ is the bin $(j, p)$ of $Y$, and histogram $Z=\left\{z_{j p}:(j, p) \in \mathcal{I}\right\}$, where $z_{j p}$ is the bin $(j, p)$ of $Z$. To compare the two histograms using EMD- $L_{1}, Y$ and $Z$ are first normalised to two unit masses (i.e., $\sum_{j, p} p_{j p}=1$ and $\sum_{j, p} q_{j p}=1$, where $p_{j p}$ and $q_{j p}$ denote the normalised masses of the earth on the bin $(j, p)$ of the histogram $Y$ and the bin $(j, p)$ of the histogram $Z$ respectively). EMD- $L_{1}$ is defined in (1).

$$
\text { EMD- } L_{1}(Y, Z)=\min _{F=\left\{f_{j, p ; c, d}:(j, p, c, d) \in \mathcal{J}_{1}\right\}} \sum_{\mathcal{J}_{1}} f_{j, p ; c, d}
$$

is subject to

$$
\left\{\begin{array}{l}
\sum_{\substack{c, d:(j, p, c, d) \in \mathcal{J}_{1} \\
f_{j, p ; c, d} \geq 0}}\left(f_{j, p ; c, d}-f_{c, d ; j, p}\right)=b_{j p} \quad \forall(j, p) \in \mathcal{I} \\
\forall(j, p, c, d) \in \mathcal{J}_{1},
\end{array}\right.
$$


where $b_{j p}$ is the difference between the two histograms $Y$ and $Z$ at the bin $(j, p)$, and a flow $F$ satisfying (2) is called a feasible flow which consists of a number of sub-flows $f_{j, p ; c, d}$. EMD $-L_{1}$ can be interpreted as a network flow model, where each bin $(j, p)$ is treated as a node with weight $b_{j p}$ and has eight directed flows between itself and its four neighbours. The intuition of constraint (2) is that the difference between the total flow entering any node $(j, p)$ on the network and the total flow leaving the node $(j, p)$ must equal to $b_{j p}$. The total weight associated with all the nodes is 0 (i.e., $\sum_{(j p) \in \mathcal{I}} b_{j p}=0$ ), since the two histograms $Y$ and $Z$ carry equal weights. Thus, the task of this network flow modelling of EMD- $L_{1}$ is to make all nodes bear zero weights by redistributing the weights via the flows.

EMD $-L_{1}$ has significantly simplified the original EMD from three aspects. First, reducing the number of variables from $N^{4}$ to $4 N$ as shown in (1). Second, decreasing the number of equality constraints by fifty percent. Third, converting all ground distances to ones, which is essentially important due to the elimination of the expensive computation of ground distances. Thus, each sub-flow $f_{j, p ; c, d}$ is equivalent to the respective weighted sub-flow $f_{j, p ; c, d} \times d_{j, p ; c, d}$, since the corresponding ground distance $d_{j, p ; c, d}$ is now set to one. Moreover, a tree-based algorithm was designed in [14] as an efficient discrete optimisation solver for EMD- $L_{1}$ to find a Basic Feasible (BF) solution (i.e., a spanning tree), which satisfies the constraint (2). The tree-based algorithm significantly boosts up the process of problem solving and achieves much higher efficiency than the original simplex algorithm.

\section{b) Reformulation of DoS Attack Detection Problem:}

However, it is not an easy mission to formula a network intrusion detection problem as a computer vision task. The above idea cannot be applied to an existing detection system as simple as a plug-and-play component to a computer system. Since the fact that EMD- $L_{1}$ was originally designed for object shape recognition, we cannot straightly use it on either network traffic payloads or network flow statistics. To achieve the task, reformulation of the existing detection system needs to be performed to fill the gap between EMD- $L_{1}$ and the ordinary detection. In this study, for instance, the ordinary network traffic records are converted into a kind of format that is used to represent images. In other words, a network traffic record, such as the observation $x_{i_{P r}}=\left[\begin{array}{lllll}f_{1_{P r}}^{i} & f_{2_{P r}}^{i} & \cdots & f_{k_{P r}}^{i}\end{array}\right]^{T}(1 \leq$ $i \leq n$ ) shown in Section III-A5, needs to be rationally transformed from the original one-dimensional feature vector into a new two-dimensional feature matrix. Two-dimensional feature matrix is the common presentation for generic twodimensional images. Through the transformation, the network traffic record to be recognised by EMD- $L_{1}$ as if it is an image. Then, EMD $-L_{1}$ can be applied to measure the dissimilarity between the transformed network traffic records.

Though the transformation of a network traffic record sounds simple, it actually cannot be accomplished via a simple manipulation. The two-dimensional feature matrix must be able to reveal the correlations between the features and provide accurate presentation to the respective network traffic record. To achieve this task, we suggest applying the MCA approach discussed in Section III-A5a to convert network traffic records.
The approaches supply high quality discriminative features and facilitate the fusion of intrusion detection and computer vision. The two-dimensional Triangle Area Maps (TAMs) are taken as the images of the analysed network traffic records. The TAMs will be filled into (1) to calculate the EMD- $L_{1}$ between the observed network traffic records.

5) Feature Extraction Schemes Based on Multivariate Correlation Analysis: Raw features of inbound network traffic, such as the ones in [15] and [38], maintain plain or hidden correlations among themselves. These correlations are often overlooked in the decision making methods, which rely only on the plain information coming from the raw features. This leads to a disadvantage in detection accuracy. In addition, the occurrence of network intrusions causes changes to these multivariate correlations so that the changes can be used as metrics for identifying intrusive activities. It gives us reasons to make good use of the significant discriminative information residing in the correlations between the raw features.

Our previously proposed MCA-based scheme [13] is applied in this paper to help extract these correlations from the features. In comparison with other approaches shown in [11] and [12], this MCA approach is proven as advanced in two respective aspects (i.e., requiring only the knowledge of current observation in performing analysis, and withstanding the problem that all features being changed linearly [11]).

Given the data set $X_{P r}=\left[\begin{array}{llll}x_{1_{P r}} & x_{2_{P r}} & \cdots & x_{n_{P r}}\end{array}\right]$, the correlative information residing in the $i^{\text {th }}$ observation $x_{i_{P r}}=$ $\left[\begin{array}{llll}f_{1_{P r}}^{i} & f_{2_{P r}}^{i} & \cdots & f_{k_{P r}}^{i}\end{array}\right]^{T}(1 \leq i \leq n)$ is extracted using the TAM-based approach as follows.

a) TAM-based MCA Approach: By contrast, the TAMbased MCA approach [13] attempts to accomplish the same task from a different perspective, in which the concept of triangle area is applied to extract the geometrical correlation between the $j^{t h}$ and $p^{t h}$ features in an observation $x_{i_{P r}}$. To obtain the triangle formed involving the $j^{\text {th }}$ and $p^{\text {th }}$ features, a data transformation is engaged. The observation $x_{i_{P r}}$ is first projected on the $(j, p)$-th two-dimensional Euclidean subspace as shown in (3).

$$
y_{i, j, p}=\left[\varepsilon_{j} \varepsilon_{p}\right]^{T} x_{i_{P r}}=\left[f_{j_{P r}}^{i} f_{p_{P r}}^{i}\right]^{T},
$$

where $1 \leq i \leq n, 1 \leq j \leq k, 1 \leq p \leq k$ and $j \neq p$. Moreover, $\varepsilon_{j}=\left[\begin{array}{llll}e_{j, 1} & e_{j, 2} & \cdots & e_{j, k}\end{array}\right]^{T}$ and $\varepsilon_{p}=$ $\left[\begin{array}{llll}e_{p, 1} & e_{p, 2} & \cdots & e_{p, k}\end{array}\right]^{T}$. The elements in the vectors $\varepsilon_{j}$ and $\varepsilon_{p}$ are all zeros, except the $(j, j)$-th and the $(p, p)$-th elements whose values are ones in $\varepsilon_{j}$ and $\varepsilon_{p}$ respectively. The projected point, $y_{i, j, p}$, is located on the Cartesian coordinate system in the $(j, p)$-th two-dimensional Euclidean subspace with coordinate $\left(f_{j_{P r}}^{i}, f_{p_{P r}}^{i}\right)$. Then, on the Cartesian coordinate system, a triangle formed by the origin and the projected points of the coordinate $\left(f_{j_{P r}}^{i}, f_{p_{P r}}^{i}\right)$ on the $j$-axis and the $p$-axis is found, and whose area is defined as $\operatorname{Tr}_{j, p}^{i}=\left(\left\|\left(f_{j_{P r}}^{i}, 0\right)-(0,0)\right\|\right.$ $\left.\times\left\|\left(0, f_{p_{P r}}^{i}\right)-(0,0)\right\|\right) / 2$, where $1 \leq i \leq n, 1 \leq j \leq k$, $1 \leq p \leq k$ and $j \neq p$. In order to make a complete analysis, all possible permutations of any two distinct features in the observation $x_{i_{P r}}$ are extracted and the corresponding triangle areas are computed. A $k$-by- $k$ matrix (i.e., a triangle area map) is constructed and represented in (4).

$$
T A M^{i}=\left[T r_{j, p}^{i}\right]_{k \times k},
$$


where all the triangle areas are arranged on the map in accordance with their indexes similar to Euclidean distance map in the EDM-based MCA approach. Additionally, the values of the elements on the diagonal of the map are set to zeros (i.e., $\operatorname{Tr}_{j, p}^{i}=0$, if $j=p$ ), because we only care about the correlation between each pair of distinct features. For the data set $X$, its geometrical multivariate correlations can be represented as $X_{T A M}=\left\{T A M^{1} T A M^{2} \ldots T A M^{n}\right\}$.

\section{B. System Framework}

In this section, we deliver the complete framework of the proposed DoS attack detection system. It elaborates the detailed processes of dimensionality reduction, normal profile generation and attack recognition. The integration of the aforementioned mechanisms into the proposed system is also presented in the discussion below. Our proposed DoS attack detection system, shown in Fig. 2, is comprised of three major steps. They are Step 1: Basic Feature Generation, Step 2: Dimensionality Reduction Based on Principal Component Analysis (PCA) and Step 3: Decision Making. Output from each step is passed down to and used as input in the next step.

1) Basic Feature Generation: In this step, basic features are generated from network traffic packets captured at the destination network. Then, they are applied to construct records describing statistics for a well-defined time interval. The detailed process can be found in [15].

2) Dimensionality Reduction Based on PCA: This step performs dimensionality reduction using PCA for the training normal traffic records generated in Step 1. The detailed algorithm presented in Section III-C1 is engaged in this task. Standing out from the feature reduction techniques, our suggested dimensionality reduction algorithm does not cause loss of information by the use of PCA which seeks the optimal subspace for the best representation of the data. The selected lower dimensional feature subspace obtained in the current step is then used in both of the Training Phase and the Test Phase involved in Step 3 (i.e., Decision Marking) to reduce the computational overhead.

3) Decision Making: This step consists of Training Phase and Test Phase. The anomaly-based detection mechanism discussed in Section III-A3 is adopted in both of the phases. The detailed introduction to this step is given as follows.

In Training Phase, normal profiles are generated for various types of legitimate/normal traffic records (i.e., TCP, UDP and ICMP traffic) using the algorithm detailed in Section III-C2. The normal traffic records used in this phase are identical to the set of records involved in Step 2. In the process of generation, normal profiles are built with the data projected onto the selected feature subspace recommended by Step 2 . The generated normal profiles (Pro) are stored in the database and are to be used in attack detection.

In Test Phase, the sample-by-sample detection mechanism discussed in Section III-A2 and the computer vision based attack recognition mechanism described in Section III-A4 are adopted. Images of individual tested records are generated and compared against the respective normal profiles Pro from the
Training Phase using EMD- $L_{1}$. As shown in Fig. 5, attack detection is modelled as a computer vision task, in which normal profiles are used as queries to retrieve the matched records (i.e., normal TCP, UDP and ICMP traffic records). Any unmatched images (records) are determined as attacks.

\section{Relevant Algorithms}

In this section, a series of algorithms are proposed to equip our system with the defined functionality. Detailed discussions are then presented to give insights into the ideas behind.

1) Algorithm for Dimensionality Reduction Based on Principal Component Analysis: As a linear mathematical system, PCA provides insight into the space where the given data resides. It also helps eliminate distractive noise and seek the optimal lower dimensional representation for data with a high dimensionality. The selected low dimensional feature space with an accurate representation for data makes significant contribution to accelerate the processing speed of the detection phase. PCA has been used in other earlier research work [26] [40] and has shown promising results. Therefore, we suggest an algorithm shown in Fig. 3 for dimensionality reduction based on PCA. Different from the work which applied PCA on dimensionality reduction for network packet payloads [26] and directly on attack detection [40], PCA is used in this work to determine the optimal feature subspace for a given set of network traffic records without containing packet payloads. In addition, we suggest using a cumulative-variance-based selection criterion in the feature subspace selection.

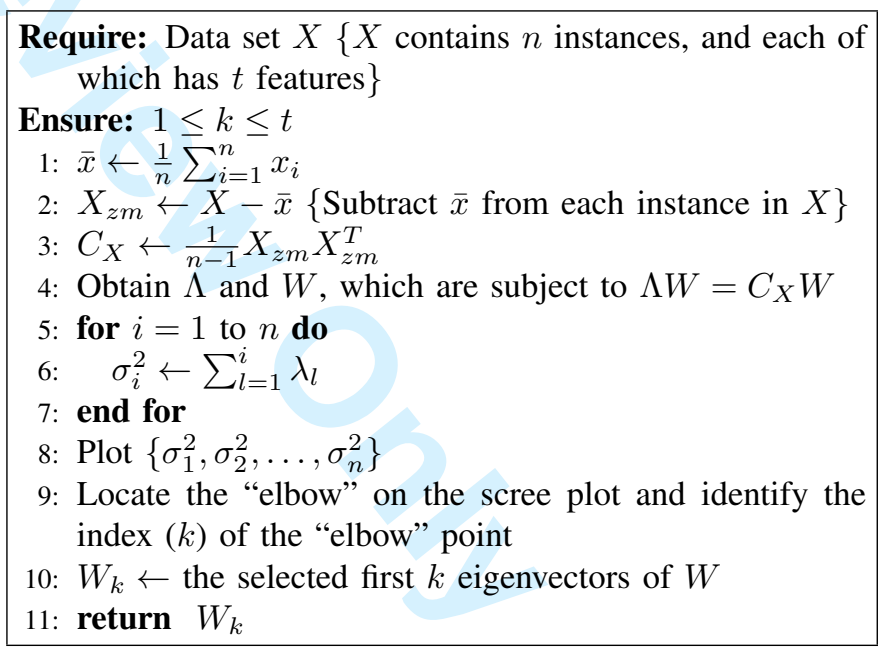

Fig. 3. Algorithm for dimensionality reduction based on the PCA.

Since PCA is driven by the idea that greater contribution on data representation comes from the eigenvectors which conserve larger variations (i.e., eigenvalues), a multivariate analysis is performed to reveal the importance of the eigenvectors in a data space to which the interested data belongs. The analysis involves a transformation converting the interested data into a new orthonormalised coordinate system, where the axes indicate the directions of the eigenvectors and the data is maximally linearly decorrelated.

In Fig. 3, the algorithm for dimensionality reduction is proposed to analyse the feature space of a given data set 


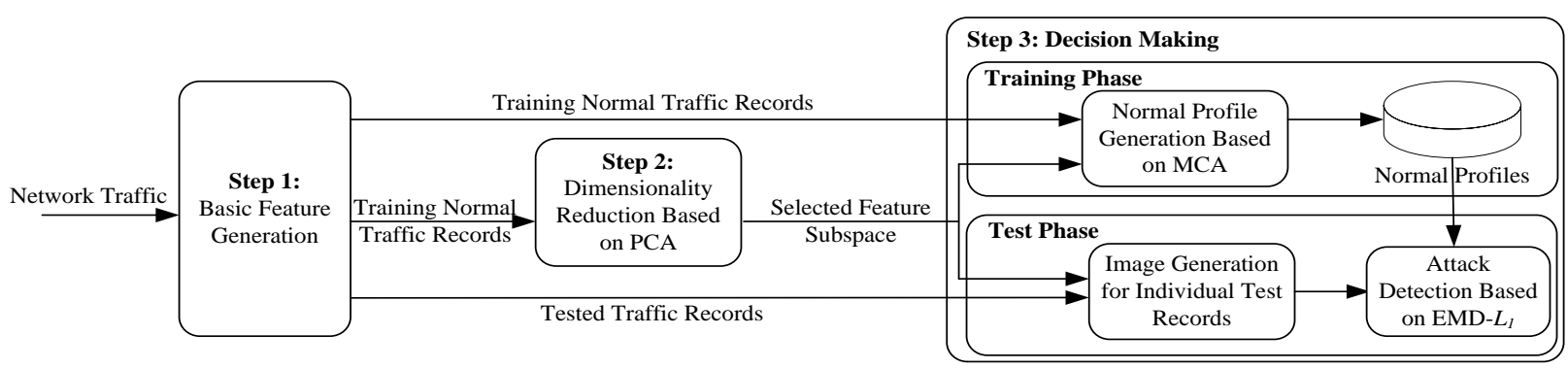

Fig. 2. Framework of our proposed denial-of-service attack detection system

$X=\left[\begin{array}{llll}x_{1} & x_{2} & \cdots & x_{n}\end{array}\right]$, where $x_{i}=\left[\begin{array}{llll}f_{1}^{i} f_{2}^{i} & \cdots & f_{t}^{i}\end{array}\right]^{T}(1 \leq i \leq n)$ denotes the $i^{t h}$ observation with $t$ features. Zero-mean normalisation is first conducted on the data set for all the observations to make the PCA work properly. The zero-mean data set is represented by $X_{z m}=\left[\left(x_{1}-\bar{x}\right)\left(x_{2}-\bar{x}\right) \cdots\left(x_{n}-\bar{x}\right)\right]$, in which $\bar{x}=\frac{1}{n} \sum_{i=1}^{n} x_{i}$. Then, the principal components (i.e., eigenvectors) are obtained by performing eigen decomposition on the sample covariance matrix $C_{X}=\frac{1}{n-1} X_{z m} X_{z m}^{T}$. The $C_{X}$ is then decomposed into a matrix $W$ and a diagonal matrix $\Lambda$. The two matrices satisfy the condition that $\Lambda W=C_{X} W$. $\Lambda$ and $W$ are sorted in descending order against the variance associated to each component. The columns of the matrix $W$ stand for the eigenvectors (i.e., the principal components) of the covariance matrix $C_{X}$, and the elements along the diagonal of the matrix $\Lambda$ are the ranked eigenvalues associated with the corresponding eigenvectors in the matrix $W$.

To determine the optimal number of principal components to be retained based on the analysis results from the PCA, a cumulative-variance-based selection criterion is applied. $\mathrm{Cu}$ mulative variance $\sigma_{i}^{2}$ is computed with an increment of one as described in lines 5 to 7 of Fig. 3 and plotted on the screen. The "elbow" point on the up-slope plot is located to determine the first $k$ most influential components. The motivation behind this assumption is that the cumulative variance increases rapidly until the "elbow" point, and the curve becomes flat beyond the point. This infers that the principal components beyond the "elbow" point retain very small variances and are not important to the representation of the data. An example will be given in Section IV-B1 to demonstrate how cumulative variance plot works. Then, the selected $k(1 \leq k \leq t)$ principal components, namely the eigenvectors in matrix $W$ which are associated with the first $k$ largest eigenvalues, provide the best presentation for the original data set and reduce the dimensionality of the original data space from $t$ to $k$. Finally, once the value of $k$ is settled, the optimal feature subspace will be obtained and denoted by $W_{k}$.

2) Algorithm for Normal Profile Generation Based on $M C A$ : Profiles of legitimate network traffic behaviours are core components to an anomaly-based detection system. Accurate characterisation to network traffic behaviours is essential and affects the detection performance of our proposed system directly. The algorithm for normal profile generation is elaborated in Fig. 4. The TAM-based MCA approach is employed in the algorithm for charactering legitimate network traffic behaviours.

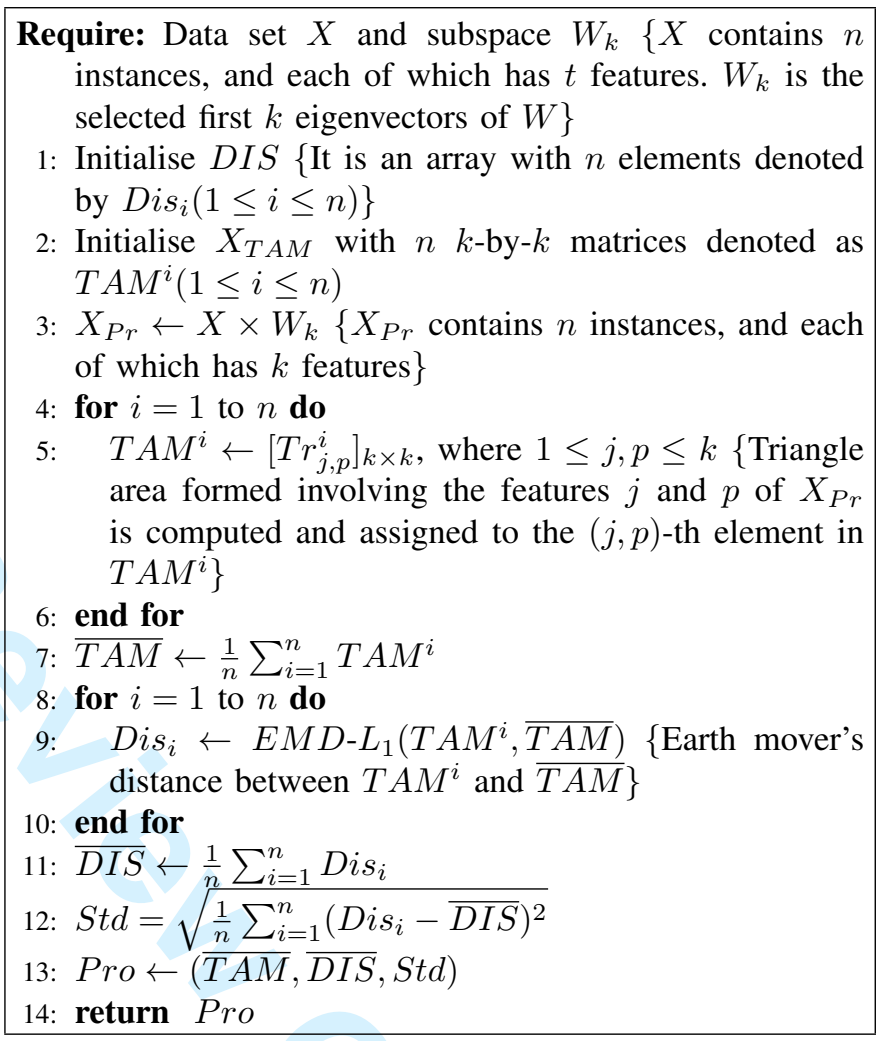

Fig. 4. Algorithm for normal profile generation based on MCA.

A normal profile is generated based on a given training data set $X$ and a selected subspace $W_{k}$. The normal profile consists of three elements, namely an image $(\overline{T A M})$ of the mean of the given training samples, the mean $(\overline{D I S})$ and the standard deviation $(S t d)$ of the earth mover's distances $\left(D i s_{i}\right)$ between individual training samples and the mean of the given training samples.

To develop the normal profile, an algorithm described in Fig. 4 is to be used. Two variables $D I S$ and $X_{T A M}$ are defined and initialised at the first place. $D I S$ is a 1-by-n array to record the earth mover's distances between the given training samples and their mean. $X_{T A M}$ is a three-dimensional ( $k$ by- $k$-by- $n$ ) matrix to store the TAMs generated for the given training samples. The previously mentioned TAM is a $k$-by- $k$ matrix and represents the image of the training sample.

The transformation of a training sample from a feature vector to an image is an important step in the process of 
normal profile generation. It bridges network traffic classification and computer vision. Since none of the computer vision techniques is initially designed for the task of network traffic classification, modification to the existing techniques or redefinition of the original problem is necessary. Thus, in this paper we redefine our network traffic classification problem as a computer vision problem, namely taking network traffic records as images and building up profile for these images. The details of the redefinition (transformation) are given below.

Dimensionality reduction is first conducted by projecting $X$ onto the selected subspace $W_{k}$ as shown in line 3 of Fig. 4 before the transformation of the given data set $X$ commences. This results in a new lower-dimensional representation $\left(X_{P r}=\left[\begin{array}{llll}x_{1_{P r}} & x_{2_{P r}} & \cdots & x_{n_{P r}}\end{array}\right]\right)$ for the given data set. The observation is now represented as $x_{i_{P r}}=$ $\left[\begin{array}{llll}f_{1_{P r}}^{i} & f_{2_{P r}}^{i} & \cdots & f_{k_{P r}}^{i}\end{array}\right]^{T}(1 \leq i \leq n)$. Then, $T A M^{i}$ is generated for each training sample using the corresponding MCA techniques discussed in Section III-A5a. The mean $\overline{T A M}$ of the image TAM is computed as shown in line 7 after the transformation is completed. Afterwards, the Earth Mover's Distance between the image of each training sample and the image of the mean of the given training samples is calculated using EMD- $L_{1}$ defined in (1) and assigned to Disi $i_{i}$. Upon the completion of measuring the Earth Mover's Distances of individual training samples to the mean, the distribution of the the Earth Mover's Distances is then estimated. The mean $(\overline{D I S})$ and the standard deviation $(S t d)$ of the EMDs $\left(D_{i s}\right)$ are computed as given in lines 11 and 12 respectively. Finally, the normal profile is built.

In order to adapt to change of a network and age out outdated data from the model, an incremental online version of our proposed detection system is introduced as follows. To compute the incremental version of EMD- $L_{1}$, we need to compute the mean $(\overline{T A M})$ for each new legitimate sample observed. The mean can be updated as $\overline{T A M}=$ $\frac{\overline{T A M} \times n+T A M^{n+1}}{n+1}=\overline{T A M}+\frac{T A M^{n+1}-\overline{T A M}}{n+1}$ when a new legitimate sample is seen [41]. This offers a means to automatically update the model and to maintain an accurate up-to-date view of normal traffic patterns.

3) Algorithm for Attack Detection Based on EMD- $L_{1}$ : The algorithm presented in Fig. 5 describes the procedure of attack recognition. To determine whether a tested sample $x_{\text {test }}$ is legitimate or intrusive, the selected feature subspace $W_{k}$, the pre-generated normal profile Pro and parameter $\alpha$ are required.

Dimensionality reduction is performed on the tested sample $x_{t e s t}$ through projecting the sample onto the selected feature subspace $W_{k}$ in order to enhance the detection speed and accuracy. Then, the transformation of the projected tested sample $x_{\text {test }}^{P r}$ to an image is conducted. The image is matched against the pre-determined query (i.e., the normal profile Pro). The similarity between the image (i.e., $T A M_{\text {test }}$ ) of the tested sample and the mean image (i.e., $\overline{T A M}$ ) from the provided normal profile Pro is measured using the EMD- $L_{1}$ and assigned to Distest.

The tested sample is finally classified as an attack or a normal record using the criterion depicted in line 4 of Fig. 5. The lower threshold on the left most hand side and the upper

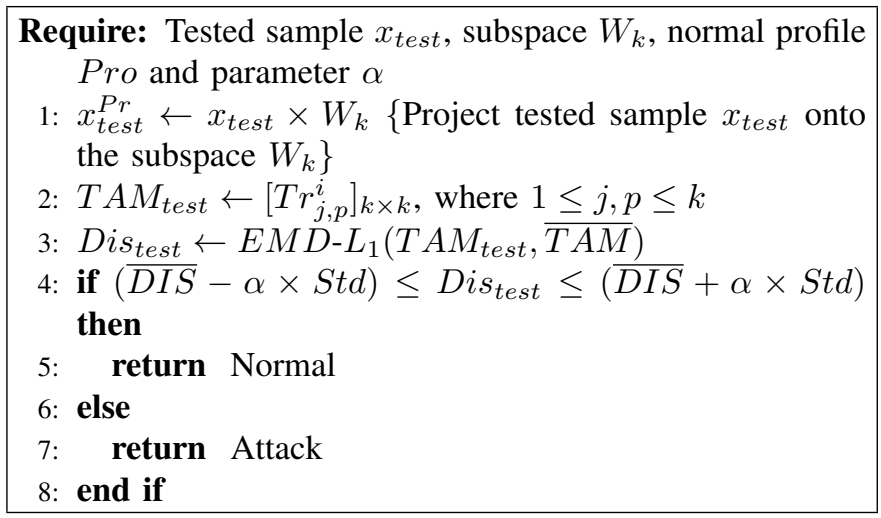

Fig. 5. Algorithm for attack detection based on EMD- $L_{1}$.

threshold on the right most hand side are both determined by three parameters $\overline{D I S}, S t d$ and $\alpha$. The parameters $\overline{D I S}$ and Std are suggested by the profile Pro developed in the phase of normal profile generation using the algorithm given in Fig. 4. The parameter $\alpha$ is ranged from 1 to 3 , and it denotes the range where network traffic records are allowed to be accepted as legitimate ones in the estimated distribution of the EMDs learnt during normal profile generation.

\section{System Evaluation}

In this section, we conduct evaluations on our proposed DoS attack detection system using KDD Cup 99 data set [15] and ISCX 2012 IDS evaluation data set [16], which are labelled benchmark data sets and publicly available on their respective online repositories.

KDD Cup 99 data set has been widely used in the domain of intrusion detection research, and remains active in many recent cutting-edge research [11] [12] [13] [42]. It has been recommended for evaluating the performance of an anomalybased IDS in detecting new intrusions. Due to the reason that the primary concern to an anomaly-based IDS is its accuracy in modelling normal traffic behaviour of a network, the age of data does not prevent a fair evaluation on the system [43]. Moreover, testing our approach using KDD Cup 99 data set contributes convincing evaluations and comparisons with other related state-of-the-art techniques [11] [12]. However, the data set has been criticised for redundant records that prevent algorithms from learning infrequent harmful records [44]. Thus, the selection of non-redundant data may apply to avoid this negative impact, but it is a labour-intensive task. Alternatively, algorithms innately withstand the problem are more desirable. As one of this kind, the underlying algorithms of our proposed DoS attack detection system are immune to the problem because its profiles are built purely based on legitimate network traffic. Therefore, the aforementioned problem introduced by the redundant data can be avoided in our evaluations.

During the evaluations, the 10 percent labelled data subset of KDD Cup 99 data set is used, where five different types of DoS attacks (Teardrop, Smurf, Pod, Neptune and Land attacks) and three types of legitimate traffic (TCP, UDP and ICMP traffic) are available. All records of the above mentioned 
network traffic from the 10 percent labelled data subset are first extracted. Then, they are further categorised into six groups according to their labels. The specific numbers of the filtered records can be found in [47]

Another evaluation data set, ISCX 2012 IDS evaluation data set, was generated from a testbed, systemically designed by the Information Security Centre of Excellence at the University of New Brunswick. The data set is intended to overcome the technical issues in other IDS evaluation data sets, and to provide network traces capturing up-to-date legitimate and intrusive network behaviours and patterns [16]. This data set consists of seven days' capturing with overall 2,450,324 traffic flows. During the evaluations, Distributed Denial of Service (DDoS) attack traffic from Tuesday's network trace is used. It contains 8,720 attack traffic flows. As such, the effectiveness of our detection system on modern traffic can be evaluated.

\section{A. Evaluation Matrices}

Four metrics, namely True Negative Rate (TNR), Detection Rate (DR), False Positive Rate (FPR) and Accuracy (i.e. the proportion of the overall samples which are classified correctly), are used to quantitatively estimate the performance of our proposed system.

\section{B. Evaluations on Detection Performance}

Ten-fold cross-validations are conducted to evaluate the performance of our proposed DoS attack detection system. We randomly select 70 percent of the filtered records from 10 percent labelled data subset of KDD Cup 99 data set to form an evaluation data set $A$, and select 70 percent of the DDoS attack traffic flows from Tuesday's network trace as well as normal traffic to form an evaluation data set $B$. This helps avoid the bias hiding in the sequential data affecting the normal profile generation and the detection performance of the proposed system. The evaluation results are reported in Tables II and III, which illustrate the trade-off between the FPR and DR as well as Accuracy again different Thresholds.

Since DDoS attacks rely on overwhelming traffic to compromise a target machine, network traffic seen at an aggregation point better reflects the behaviours of attack instances. As discussed in Section III-B, an IDS is recommended to position at an entry point to a protected local network to monitor and detect anomaly traffic patterns. Thus, the detection accuracy of the detection system on aggregate traffic reflects its detection capability. The detailed evaluations to our proposed detection system are presented as follows.

1) Dimensionality Reduction: Analysis on the selected filtered legitimate (Normal) traffic is conducted using the algorithm given in Fig. 3 to help determine the optimal feature subspace for data representation for the entire training data set. Three feature subspaces are chosen with respect to normal TCP, UDP and ICMP traffic. The selected feature subspaces are used in Training Phase (Section IV-B2) and the Test Phase (Section IV-B3) to supply with accurate representation for all records. The new lower dimensional representations of the records are used to train and to test the proposed DoS detection system. As proposed in Section III-C1, we apply the
TABLE I

THE Numbers OF PRINCIPLE COMPONENTS USING IN THE TRAINING AND TEST FOr VARIOUS Network TRAFFiC FROM KDD CUP 99 DATA SET

\begin{tabular}{|c|c|c|c|c|c|c|c|}
\hline Type of Traffic & TCP & \multicolumn{3}{|c|}{ UDP } & \multicolumn{3}{c|}{ ICMP } \\
\hline \hline No. of PCs & 3 PCs & 5 PCs & 6 PCs & 7 PCs & 3 PCs & 4 PCs & 5 PCs \\
\hline
\end{tabular}

plot of accumulative variances in the election of the optimal feature subspaces. The up-slope on the plot indicates the potential optimal subspace for data representation. Thus, we can eliminate those less important PCs and retain only the first a few critical PCs to form a new low dimensional feature space.

To determine the number of critical PCs to be retained for various types of network traffic in our evaluators, the accumulative variance plots for normal TCP, UDP and ICMP traffic extracted from KDD Cup 99 data set are shown in Figs. 6a-6c respectively. The horizontal axes of the figures stand for the number of PCs, and the vertical axes of the figures represent the accumulative variances with respect to the numbers of PCs shown on the horizontal axes. The upslopes on the plots for TCP, UDP and ICMP traffic are found lying at the first two PCs, the first six PCs and the first four PCs respectively. The same result is seen in ISCX 2012 IDS evaluation data set. However, these numbers are not always practicable, and the best performance may be achieved around these numbers. For instance, using only the first two PCs to represent the TCP traffic is not applicable in our detection system. This is because the maps (i.e., TAM) constructed using only two features are always identical for all records after normalisation. Hence, we will choose the first three PCs instead of the first two PCs.

2) Training Phase: In the Training Phase of the Decision Marking (Step 3) shown in Fig. 2, profiles are generated with respect to various types (i.e., TCP, UDP and ICMP) of Normal traffic records. Moreover, as the plots of the accumulative variances only suggest the preliminary results, we need to conduct further selection based on the suggestion from the preliminary outcomes from Section IV-B1. In this work, we test three sets of PCs for each types of traffic, except TCP traffic. According to the reason given in Section IV-B1, we decide to use the first three PCs for TCP traffic only. The numbers of PCs used in the further selection are given in Table I. Normal profiles are built with respect to the chosen feature subspaces (i.e., the aforementioned numbers of PCs). Then, the generated normal profiles are utilised in the Test Phase.

3) Test Phase: During the Test Phase of the Decision Marking shown in Fig. 2, we test our proposed detection system against both the Normal records and the attack records in the evaluation data set. The thresholds with respect to different normal profiles are determined given the parameter $\alpha$ varying from 1 to 3 with an increment of 0.5 . The tests run against the various sets of PCs (i.e., the selected lower dimensional subspaces) shown in Table I. The best performance is achieved on the first three PCs for TCP traffic and the first five PCs for both UDP and ICMP traffic. Tables II and III present the corresponding experimental results for our 


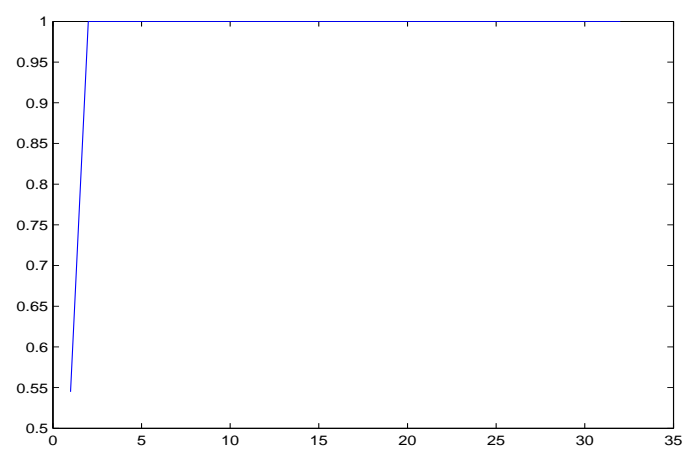

(a) Accumulative variance plot for TCP traffic

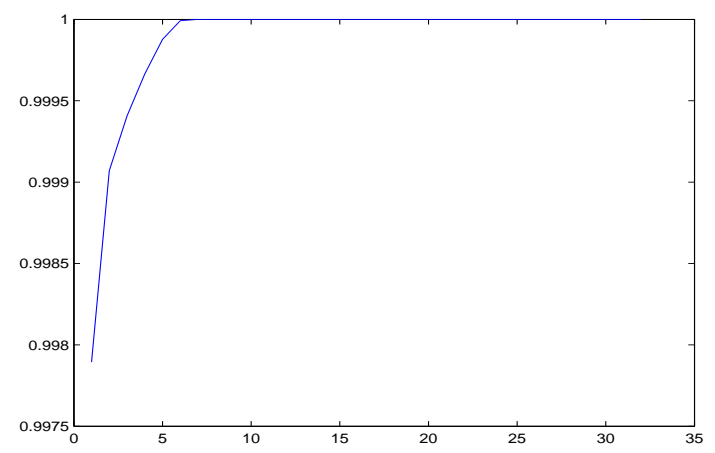

(b) Accumulative variance plot for UDP traffic

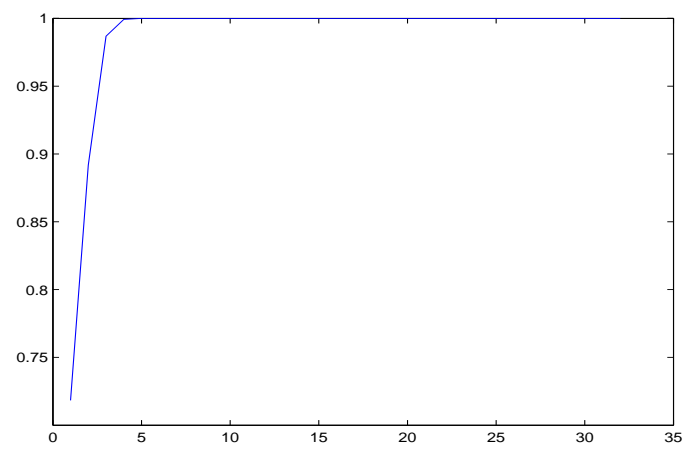

(c) Accumulative variance plot for ICMP traffic

Fig. 6. Accumulative variance plots for TCP, UDP and ICMP traffic from KDD Cup 99 data set

proposed detection system on KDD Cup 99 data set and ISCX 2012 IDS evaluation data set respectively.

TABLE II

False Positive Rates, Detection Rates and Accuracies ACHIEVEd B Y THE PRoposed System Based ON KDD CUP 99 DATA SET

\begin{tabular}{|l|l|l|l|l|l|}
\hline \multirow{2}{*}{} & \multicolumn{5}{|c|}{ Threshold } \\
\cline { 2 - 6 } & $1 \sigma$ & $1.5 \sigma$ & $2 \sigma$ & $2.5 \sigma$ & $3 \sigma$ \\
\hline \hline FPR & $1.93 \%$ & $1.19 \%$ & $0.63 \%$ & $0.60 \%$ & $0.58 \%$ \\
\hline DR & $100.00 \%$ & $99.83 \%$ & $99.68 \%$ & $99.68 \%$ & $93.35 \%$ \\
\hline Accuracy & $99.95 \%$ & $99.81 \%$ & $99.67 \%$ & $99.67 \%$ & $93.50 \%$ \\
\hline
\end{tabular}

As shown in Tables II and III, the threshold controls the degree of the dissimilarity, which is accepted by the system, between a test object and the respective learnt normal profile. If the dissimilarity is beyond the determined threshold, the test object is classified as an attack. On one hand, it can be seen clearly from Tables II and III that a better FPR is achieved when a greater threshold is accepted. On the other hand, greater thresholds produce lower DRs.

To provide a visualisation for the trade-off between Accuracy and Threshold, Fig. 7 is given below. The proposed detection system enjoys promising performance on KDD Cup 99 data set with $99.95 \%$ accuracy when the threshold is set to $1 \sigma$. The accuracy of the both systems declines stably to $99.67 \%$ at the threshold of $2.5 \sigma$. After this point, the proposed detection system based on TAM drops significantly to $93.50 \%$.

When evaluating using ISCX 2012 IDS evaluation data set, the proposed detection system achieves slightly lower but remaining desirable accuracy (i.e., 90.12\%) at the threshold of $1 \sigma$. However, the accuracy falls down to $51.54 \%$ when the threshold sits at $1.5 \sigma$. While the threshold reaches to $3 \sigma$, the accuracy drops to its minimum $46.15 \%$.

Although our proposed detection system does not perform as good as on KDD Cup 99 data set, these results verify that it is capable of coping with current networks (e.g., ISCX 2012 IDS evaluation data set). The ever-evolving complex network architectures and sophisticated network intrusion skills account for this degradation in detection accuracy on ISCX 2012 IDS evaluation data set. Employing a Collaborative IDS (CIDS) framework, in which standalone fellow IDSs cooperate with each other to share information and to construct a complete attack diagram of an entire protected network, could help improve detection accuracy [45]. However, this is out of the scope of the work presented in this paper and will be studied in our feature research.

\section{Comparison of Performance}

To show a clearer picture that how our proposed DoS attack detection system performs, we, on one hand, make comparisons with three state-of-the-art detection systems on their detection accuracy achieved on KDD Cup 99 data set in this section. The best performance of these systems is selected and shown in Table IV. The comparison results illustrate that our proposed detection system based on EMD in cooperation with TAM-based MCA achieves $99.95 \%$ accuracy on KDD Cup 99 data set, which considerably outperforms the two other systems and remains consistent with one of my previous systems in terms of detection accuracy.

TABLE III

False Positive Rates, Detection Rates and AcCuracies ACHIEVED B T THE PROPOSED SYSTEM BASED ON ISCX 2012 IDS EVALUATION DATA SET

\begin{tabular}{|l|l|l|l|l|l|}
\hline \multirow{2}{*}{} & \multicolumn{5}{|c|}{ Threshold } \\
\cline { 2 - 6 } & $1 \sigma$ & $1.5 \sigma$ & $2 \sigma$ & $2.5 \sigma$ & $3 \sigma$ \\
\hline \hline FPR & $7.92 \%$ & $4.75 \%$ & $3.33 \%$ & $2.00 \%$ & $1.25 \%$ \\
\hline DR & $90.04 \%$ & $49.82 \%$ & $49.64 \%$ & $49.48 \%$ & $44.09 \%$ \\
\hline Accuracy & $90.12 \%$ & $51.54 \%$ & $51.41 \%$ & $51.31 \%$ & $46.15 \%$ \\
\hline
\end{tabular}


TABLE IV

Performance Comparisons with Different Detection Approaches on KDD Cup 99 Data Set

\begin{tabular}{|l|l|l|l|l|}
\hline & $\begin{array}{l}\text { Network intrusion detection based } \\
\text { on covariance feature space [11] } \\
\text { (Threshold approach with 4D prin- } \\
\text { ciple and Cov_len3_150) }\end{array}$ & $\begin{array}{l}\text { Triangle area based } \\
\text { nearest neighbours } \\
\text { approach [12] }\end{array}$ & $\begin{array}{l}\text { A system for DoS attack detec- } \\
\text { tion using TAM-based MCA } \\
\text { [13] (Normalized data, Thresh- } \\
\text { old }=1.5 \sigma)\end{array}$ & $\begin{array}{l}\text { The proposed DoS attack } \\
\text { TAM and EMD (Threshold } \\
=1 \sigma)\end{array}$ \\
\hline \hline Accuracy & $97.89 \%$ & $92.15 \%$ & $99.95 \%$ & $99.95 \%$ \\
\hline
\end{tabular}

TABLE V

False Positive Rates And Detection Rates AChieved by the APPROACHES REPORTED IN [46] ON ISCX 2012 IDS EVALUATION DATA

\begin{tabular}{|l|l|l|l|l|}
\hline \multirow{2}{*}{} & \multicolumn{4}{|c|}{ Reported Detection Approaches } \\
\cline { 2 - 5 } & NB & Bagged-NB & Boosted-NB & AMGA2-NB \\
\hline \hline FPR & $64.5 \%$ & $62.2 \%$ & $64.5 \%$ & $4.8 \%$ \\
\hline DR & $98.4 \%$ & $98.4 \%$ & $98.4 \%$ & $92.7 \%$ \\
\hline
\end{tabular}

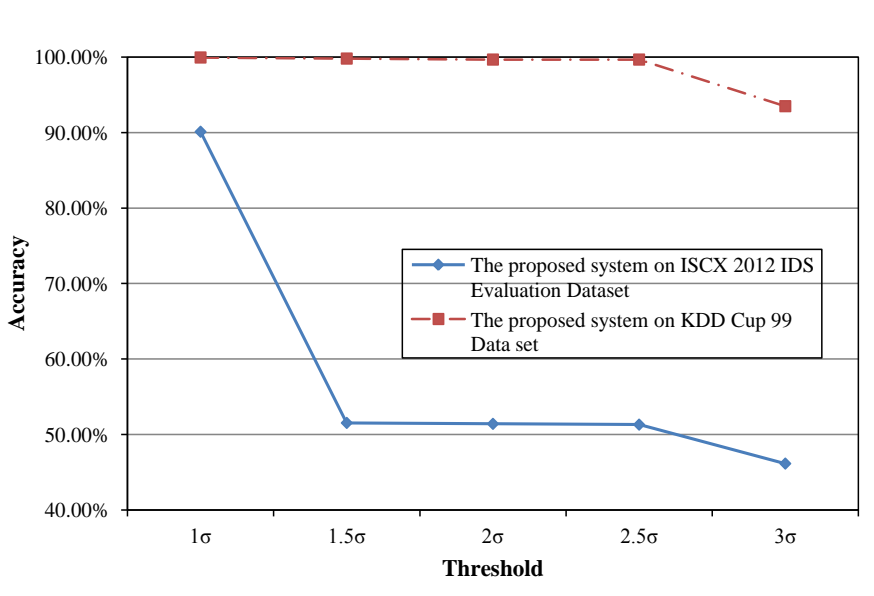

Fig. 7. Correlation between accuracy and threshold
Those two systems, namely covariance feature space based network intrusion detection system [11] and network intrusion detection using triangle-area-based nearest neighbours approach [12], achieve $97.89 \%$ and $92.15 \%$ accuracy on KDD Cup 99 data set respectively. The system that we previously developed, namely a system for DoS attack detection using TAM-based MCA [13], maintains $99.95 \%$ detection accuracy on KDD Cup 99 data set.

On the other hand, we compare the detection performance of our proposed detection system on ISCX 2012 IDS Evaluation data set with those achieved by four other detection pproaches (e.g., Naive Bayes (NB), Bagged-NB, BoostedB and AMGA2-NB) discussed [46]. The reported FPRs DRs of these four detection approaches are recapped in V. Although they achieve higher DRs than our proposed system, their detection rates are not reported for S attacks only but also take other attacks into account. So, confirm if these approaches do perform better than proposed system on DDoS attack detection. In addition, it is a nour detection approaches KDD Cup that is higher than $70 \%$ on DoS attacks from four approaches in fact outperforms our proposed DoS attack detection system.

\section{SET (INCLUDING DDOS ATTACKS AND OTHER ATTACKS)}

Although, in comparison with our previous work shown in [13], the proposed DoS attack detection system does not show a significant advance in terms of detection accuracy, it is worth noticing that the proposed system easily achieves the equal performance requiring significantly less information (i.e., fewer features involved in analysis and detection). This reduces the computational overhead.

\section{AnAlysis on COMPUtational COMPLEXity AND TIME CosT}

In this section, we conduct an analysis on the computational complexity of our proposed detection system in two ways (i.e., the complexity of the feature extraction and the complexity of the detection) and on its time cost.

As discussed in Section III-A5a, during feature extraction, triangle areas formed involving possible combinations of two distinct features in a traffic record need to be computed when processing the TAM-based MCA approach, which delivers a computational complexity of $O\left(\mathrm{~m}^{2}\right)$ due to the fact that $\mathrm{m}^{2}$ triangle areas are generated and are used to construct a TAM as well. However, as the TAM is a symmetric matrix and the elements along the main diagonal of the matrix are zeros, the numbers of the computation of this MCA approach can be reduced by more than $50 \%$ when it is put into practise. Whereas, this does not reduce their computational complexities. In attack detection, EMD- $L_{1}$ [14] is applied. As explained in Section III-A4, EMD- $L_{1}$ incurs a complexity of $O\left(N^{2}\right)$, where $N=m^{2}$ is the number of elements within a TAM. Thus, taking the computational complexities of the feature extraction and the detection into account, the overall computational complexity of the proposed detection system is $O\left(m^{2}\right)+O\left(m^{4}\right)=O\left(m^{4}\right)$.

Network intrusion detection system based on covariance feature space [11] incurs a computational complexity of $O\left(2 n \times \frac{m \times(m+1)}{2}\right)=O\left(n m^{2}\right)$ in data preprocessing, where $n$ is the number of sequential samples in a group and $m$ is the number of physical features of a sample. In attack detection, the observed covariance matrix of a group of sequential samples needs to be compared with all $l$ known classes/clusters. Therefore, it has a computational complexity of $O\left(l \mathrm{~m}^{2}\right)$. The overall computational complexity of the network intrusion detection system based on covariance feature space is $O\left(n m^{2}\right)+O\left(l m^{2}\right)=O\left(l m^{2}\right)$

Triangle-area-based nearest neighbours approach [12] has an overall computational complexity of $O\left(m l^{2}\right)+O\left(l^{2} n^{2}\right)$, in which $O\left(m l^{2}\right)$ and $O\left(l^{2} n^{2}\right)$ are complexities of the data preprocessing and the attack detection respectively ( $m$ is the number of features in a traffic record, $l$ is the number of clusters used in generating triangle areas and $n$ is the number 
TABLE VI

COMPUTATIONAL COMPlexities OF DiFFERENT STATE-OF-THE-ART DETECTION APPROACHES

\begin{tabular}{|l|l|l|}
\hline $\begin{array}{l}\text { The proposed } \\
\text { detection system }\end{array}$ & $\begin{array}{l}\text { Network intrusion de- } \\
\text { tection based on covari- } \\
\text { ance feature space [11] }\end{array}$ & $\begin{array}{l}\text { Triangle area based } \\
\text { nearest neighbours } \\
\text { approach [12] }\end{array}$ \\
\hline \hline$O\left(m^{4}\right)$ & $O\left(l m^{2}\right)$ & $O\left(l^{2} n^{2}\right)$ \\
\hline
\end{tabular}

of training samples). The complexity can be rewritten as $O\left(l^{2} n^{2}\right)$.

In general, our proposed detection system can achieve comparable computational complexity to the two other approaches. Table VI is provided to summarise the computational complexities of the above discussed approaches. Moreover, time cost is discussed to demonstrate the capability of our proposed detection system in data processing. Approximately 59,738 traffic records can be proceeded per second by our DoS attack detection system in cooperation with TAM-based MCA.

\section{CONCLUSION}

This paper has proposed a DoS attack detection system which is equipped with our previously developed MCA technique and the EMD- $L_{1}$. The former technique helps extract the correlations between individual pairs of two distinct features within each network traffic record and offers more accurate characterisation for network traffic behaviours. The latter technique facilitates our system to be able to effectively distinguish both known and unknown DoS attacks from legitimate network traffic.

Evaluation has been conducted using the KDD Cup 99 data set and ISCX 2012 IDS evaluation data set to verify the effectiveness and performance of the proposed DoS attack detection system. The results have revealed that our detection system achieves maximum $99.95 \%$ detection accuracy on KDD Cup 99 data set and $90.12 \%$ detection accuracy on ISCX 2012 IDS evaluation data set. It outperforms three state-of-theart approaches on KDD Cup 99 data set and shows advantages over the four NB-based detection approaches on ISCX 2012 IDS evaluation data set. Moreover, we have analysed the computational complexity of the proposed detection system, which achieves comparable performance in comparison with state-of-the-art approaches. The time cost analysis shows that the proposed detection system is able to cope with high speed network segments.

As our future research focus, a new CIDS will be invented based on the detection approach proposed in this article. The new CIDS will contribute an enhancement to the security of the increasingly important Cloud computing environments with its capability of handling sophisticated cooperative intrusions.

\section{REFERENCES}

[1] Neustar, "2014 - Neustar Annual DDoS Attacks and Impact Report," http://www.neustar.biz/resources/whitepapers/ddos-protection/2014annual-ddos-attacks-and-impact-report.pdf, 2014.
[2] Prolexic. "Prolexic Issues High Alert Threat Advisory for DNS Flooder DDoS Attack Toolkit," 5 August 2014; http://www.prolexic.com/newsevents-pr-threat-advisory-ddos-dns-flooder.html.

[3] R. Broadhurst, and L. C. Chang, "Cybercrime in Asia: Trends and Challenges," Handbook of Asian Criminology, J. Liu, B. Hebenton and S. Jou, eds., pp. 49-63: Springer New York, 2013.

[4] C. Douligeris, and A. Mitrokotsa, "DDoS attacks and defense mechanisms: classification and state-of-the-art," Computer Networks, vol. 44, no. 5, pp. 643-666, 2004.

[5] M. Bando, N. S. Artan, and H. J. Chao, "Scalable Lookahead Regular Expression Detection System for Deep Packet Inspection," Networking, IEEE/ACM Transactions on, vol. 20, no. 3, pp. 699-714, 2012.

[6] A. Bremler-Barr, and Y. Koral, "Accelerating Multipattern Matching on Compressed HTTP Traffic," Networking, IEEE/ACM Transactions on, vol. 20, no. 3, pp. 970-983, 2012.

[7] M. A. Jamshed, J. Lee, S. Moon, I. Yun, D. Kim, S. Lee, Y. Yi, and K. Park, "Kargus: a highly-scalable software-based intrusion detection system," in Proceedings of the 2012 ACM conference on Computer and communications security, Raleigh, North Carolina, USA, 2012, pp. 317328.

[8] D. E. Denning, "An Intrusion-Detection Model," Software Engineering, IEEE Transactions on, vol. 13, no. 2, pp. 222-232, 1987.

[9] A. Patcha and J. M. Park, "An Overview of Anomaly Detection Techniques: Existing Solutions and Latest Technological Trends," Computer Networks, vol. 51, pp. 3448-3470, 2007.

[10] M. Thottan, and C. Ji, "Anomaly detection in IP networks," Signal Processing, IEEE Transactions on, vol. 51, no. 8, pp. 2191-2204, 2003.

[11] S. Jin, D. S. Yeung, and X. Wang, "Network intrusion detection in covariance feature space," Pattern Recognition, vol. 40, no. 8, pp. 21852197, 2007.

[12] C. F. Tsai and C. Y. Lin, "A Triangle Area Based Nearest Neighbors Approach to Intrusion Detection," Pattern Recognition, vol. 43, pp. 222$229,2010$.

[13] Z. Tan, A. Jamdagni, X. He, P. Nanda, and R. Liu, "A System for Denialof-Service Attack Detection Based on Multivariate Correlation Analysis," Parallel and Distributed Systems, IEEE Transactions on, vol. 25, no. 2, pp. 447-456, 2014.

[14] H. Ling, and K. Okada, "An Efficient Earth Mover's Distance Algorithm for Robust Histogram Comparison," Pattern Analysis and Machine Intelligence, IEEE Transactions on, vol. 29, no. 5, pp. 840-853, 2007.

[15] S. J. Stolfo, W. Fan, W. Lee, A. Prodromidis, and P. K. Chan, "Costbased modeling for fraud and intrusion detection: results from the JAM project," in DARPA Information Survivability Conference and Exposition, 2000. DISCEX '00. Proceedings, 2000, pp. 130-144 vol.2.

[16] A. Shiravi, H. Shiravi, M. Tavallaee, and A. A. Ghorbani, "Toward developing a systematic approach to generate benchmark datasets for intrusion detection," computers \& security, vol. 31, no. 3, pp. 357-374, 2012.

[17] E. Levy, "Approaching zero attack trends," Security \& Privacy, IEEE , vol.2, no.4, pp. 65- 66, July-Aug. 2004.

[18] K. Lee, J. Kim, K. H. Kwon, Y. Han, and S. Kim, "DDoS attack detection method using cluster analysis," Expert Systems with Applications, vol. 34, no. 3, pp. 1659-1665, 2008.

[19] A. Tajbakhsh, M. Rahmati, and A. Mirzaei, "Intrusion detection using fuzzy association rules," Applied Soft Computing, vol. 9, no. 2, pp. 462469, 2009.

[20] S. Mukkamala, A. H. Sung, and A. Abraham, "Intrusion detection using an ensemble of intelligent paradigms," Journal of Network and Computer Applications, vol. 28, no. 2, pp. 167-182, 2005.

[21] J. Yu, H. Lee, M.-S. Kim, and D. Park, "Traffic flooding attack detection with SNMP MIB using SVM," Computer Communications, vol. 31, no. 17, pp. 4212-4219, 2008.

[22] W. Haining, Z. Danlu, and K. G. Shin, "Change-point monitoring for the detection of DoS attacks," Dependable and Secure Computing, IEEE Transactions on, vol. 1, no. 4, pp. 193-208, 2004.

[23] S. S. Kim, and A. L. N. Reddy, "Statistical Techniques for Detecting Traffic Anomalies Through Packet Header Data," Networking, IEEE/ACM Transactions on, vol. 16, no. 3, pp. 562-575, 2008.

[24] G. Thatte, U. Mitra, and J. Heidemann, "Parametric Methods for Anomaly Detection in Aggregate Traffic," Networking, IEEE/ACM Transactions on, vol. 19, no. 2, pp. 512-525, 2011.

[25] S. T. Sarasamma, Q. A. Zhu, and J. Huff, "Hierarchical Kohonenen net for anomaly detection in network security," Systems, Man, and Cybernetics, Part B: Cybernetics, IEEE Transactions on, vol. 35, no. 2, pp. 302-312, 2005. 
[26] A. Jamdagni, Z. Tan, X. He, P. Nanda, and R. P. Liu, "RePIDS: A multi tier Real-time Payload-based Intrusion Detection System," Computer Networks, vol. 57, no. 3, pp. 811-824, 2013.

[27] K. Seong Soo, and A. L. N. Reddy, "A study of analyzing network traffic as images in real-time," in INFOCOM 2005. 24th Annual Joint Conference of the IEEE Computer and Communications Societies. Proceedings IEEE, 2005, pp. 2056-2067 vol. 3.

[28] R. Fontugne, T. Hirotsu, and K. Fukuda, "An image processing approach to traffic anomaly detection," in Proceedings of the 4th Asian Conference on Internet Engineering, Pratunam, Bangkok, Thailand, 2008, pp. 17-26.

[29] S. S. Kim, and A. N. Reddy, "Image-based anomaly detection technique: algorithm, implementation and effectiveness," Selected Areas in Communications, IEEE Journal on, vol. 24, no. 10, pp. 1942-1954, 2006.

[30] Y. Rubner, C. Tomasi, and L. J. Guibas, "A metric for distributions with applications to image databases," in Computer Vision, 1998. Sixth International Conference on, 1998, pp. 59-66.

[31] Y. Rubner, C. Tomasi, and L. Guibas, "The Earth Mover's Distance as a Metric for Image Retrieval," International Journal of Computer Vision, vol. 40, no. 2, pp. 99-121, 2000/11/01, 2000.

[32] F. L. Hitchcock, "The Distribution of a Product from Several Sources to Numerous Localities ," Journal of mathematics and physics, vol. 20, pp. 224-230, 1941.

[33] F. S. Hillier, and G. J. Lieberman, Introduction to mathematical programming: McGraw-Hill, 1995.

[34] K. Grauman, and T. Darrell, "Fast contour matching using approximate earth mover's distance," in Computer Vision and Pattern Recognition, 2004. CVPR 2004. Proceedings of the 2004 IEEE Computer Society Conference on, 2004, pp. I-220-I-227 Vol.1.

[35] Q. Zhao, Z. Yang, and H. Tao, "Differential Earth Mover's Distance with Its Applications to Visual Tracking," Pattern Analysis and Machine Intelligence, IEEE Transactions on, vol. 32, no. 2, pp. 274-287, 2010.

[36] A. Y. Fu, W. Liu, and X. Deng, "Detecting Phishing Web Pages with Visual Similarity Assessment Based on Earth Mover's Distance (EMD)," Dependable and Secure Computing, IEEE Transactions on, vol. 3, no. 4, pp. 301-311, 2006.

[37] T.-F. Yen, and M. K. Reiter, "Are Your Hosts Trading or Plotting? Telling P2P File-Sharing and Bots Apart," in Distributed Computing Systems (ICDCS), 2010 IEEE 30th International Conference on, 2010, pp. 241252.

[38] S. T. Brugger, "Data mining methods for network intrusion detection," University of California at Davis, 2004

[39] A. Micarelli, and G. Sansonetti, "A Case-Based Approach to Anomaly Intrusion Detection," Machine Learning and Data Mining in Pattern Recognition, Lecture Notes in Computer Science P. Perner, ed., pp. 434448: Springer Berlin Heidelberg, 2007.

[40] A. Lakhina, M. Crovella, and C. Diot, "Diagnosing network-wide traffic anomalies," SIGCOMM Comput. Commun. Rev., vol. 34, no. 4, pp. 219230, 2004.

[41] D. E. Knuth, The Art of Computer Programming, Vol. 1 Fundamental Algorithms. Addison Wesley, 2nd edition, 1973.

[42] J. Z. Lei, and A. A. Ghorbani, "Improved competitive learning neural networks for network intrusion and fraud detection," Neurocomputing, vol. 75, no. 1, pp. 135-145, 2012

[43] V. Engen, J. Vincent, and K. Phalp, "Exploring discrepancies in findings obtained with the KDD Cup '99 data set," Intell. Data Anal., vol. 15, no. 2, pp. 251-276, 2011

[44] M. Tavallaee, E. Bagheri, L. Wei, and A. A. Ghorbani, "A Detailed Analysis of the KDD Cup 99 Data Set," The The Second IEEE International Conference on Computational Intelligence for Security and Defense Applications, 2009, pp. 1-6.

[45] Z. Tan, U. T. Nagar, X. He, P. Nanda, R. Liu, S. Wang, and J. Hu, "Enhancing Big Data Security with Collaborative Intrusion Detection," IEEE Cloud Computing Magazine, 2014, In Press.

[46] G. Kumar, and K. Kumar, "Design of an Evolutionary Approach for Intrusion Detection," The Scientific World Journal, Vol. 2013, pp. 1-14, 2013.

[47] Z. Tan, A. Jamdagni, X. He, P. Nanda, and R. Liu, "Denial-of-Service Attack Detection Based on Multivariate Correlation Analysis," Neural Information Processing, Lecture Notes in Computer Science B.-L. Lu, L. Zhang and J. Kwok, eds., pp. 756-765: Springer Berlin Heidelberg, 2011.

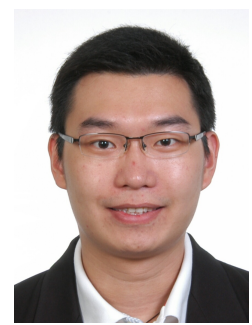

Zhiyuan Tan received his $\mathrm{PhD}$ degree from University of Technology Sydney (UTS), Australia in 2014. He is a Post-doctoral Research Fellow in the Faculty of Electrical Engineering, Mathematics and Computer Science, University of Twente, Enschede, Netherlands. He is an IEEE Member. His research interests are network security, pattern recognition, machine learning and distributed systems. The work presented in this paper was performed when Zhiyuan was a Research Associate with the School of Computing and Communications at UTS

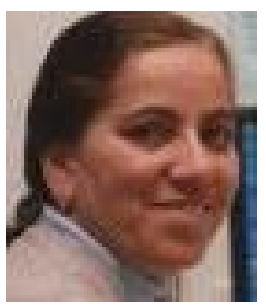

Aruna Jamdagni received her $\mathrm{PhD}$ degree from University of Technology Sydney, Australia in 2012. She is a lecturer in the School of Computing and Mathematics, University of Western Sydney (UWS), Australia, and a research member of Research Centre for Innovation in IT Services and Applications (iNEXT) at University of Technology Sydney (UTS), Australia. Her research interests include Computer and Network Security and on Pattern Recognition techniques and fuzzy set theory.

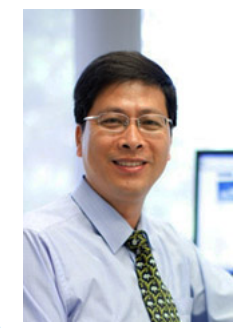

Xiangian He is a Professor of Computer Science, School of Computing and Communications. He is also Director of Computer Vision and Recognition Laboratory, the leader of Network Security Research group, and a Deputy Director of Research Centre for Innovation in IT Services and Applications (iNEXT) at the University of Technology, Sydney (UTS). He is an IEEE Senior Member. He has been awarded Internationally Registered Technology Specialist by International Technology Institute (ITI). His research pattern recognition and computer vision.

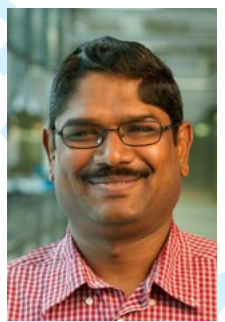

Priyadarsi Nanda is a Senior Lecturer in the School of Computing and Communications at the University of Technology, Sydney (UTS). He is also a Core Research Member at the Centre for Innovation in IT Services Applications (iNEXT) at UTS. He is an IEEE Senior Member. His research interests are in network security, network QoS, sensor networks, and wireless networks. In recent years he has been very active leading the Network Security and Applications research group at UTS. Dr Nanda has over 23 years of research and teaching experience, and has published over 50 research publications.

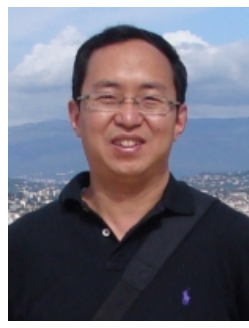

Ren Ping Liu a Principal Scientist of networking technology in CSIRO. He is also an Adjunct Professor at Macquarie University, and University of Technology, Sydney. His research interests include MAC protocol design, Markov analysis, QoS scheduling, TCP/IP internetworking, and network security. He has over 100 research publications in leading international journals and conferences. Professor Liu is a Senior Member of IEEE. He served as TPC chair, as OC co-chair, and in Technical Committee in a number of IEEE Conferences.

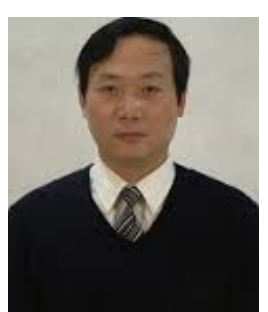

Jiankun Hu is Full Professor and Research Director of Cyber Security Lab, School of Engineering and IT, University of New South Wales at the Australian Defence Force Academy (UNSW@ADFA), Canberra, Australia. He has obtained his $\mathrm{PhD}$ in Control Engineering from Harbin Institute of Technology, China in 1993. Jiankun's main research interest is in the field of cyber security including biometrics security where he has published many papers in high-quality conferences and journals. He has served in the editorial board of up to 7 international journals and served as Security Symposium Chair of IEEE flagship conferences $\mathrm{He}$ has obtained 7 ARC (Australian Research Council) Grants and is now serving at the prestigious Panel of Mathematics, Information and Computing Sciences (MIC), ARC ERA Evaluation Committee. 


\section{RESPONSES TO REVIEWERS' COMMENTS}

NAME OF JOURNAL: IEEE Transactions on Computers

REFERENCE NUMBER: TC-2014-04-0277

Title OF PAPER: Detection of Denial-of-Service Attacks Based on Computer Vision Techniques

Authors: Zhiyuan Tan, Aruna Jamdagni, Xiangjian He, Priyadarsi Nanda, Ren Ping Liu, and Jiankun $\mathrm{Hu}$

\section{We thank all reviewers for their valuable feedback.}

Reviewers 2 and 3 recommend accepting this manuscript with NO changes. Reviewers 1 and 4 recommend a minor revision and a major revision respectively. We respond to the reviewers' comments as follows.

Responses to Reviewer 1's comments

\begin{tabular}{|c|c|c|}
\hline No. & Comments & Revisions \\
\hline 1 & $\begin{array}{l}\text { The paper proposes an interesting and } \\
\text { potentially novel approach to the DoS } \\
\text { detection issue. }\end{array}$ & We thank the reviewer's acknowledgement. \\
\hline 2 & $\begin{array}{l}\text { The Abstract mentions the evaluation of the } \\
\text { proposed approach but does not present } \\
\text { details of the actual results observed (e.g. \% } \\
\text { effectiveness and operational cost). }\end{array}$ & $\begin{array}{l}\text { We thank the reviewer's comment. As } \\
\text { suggested, the actual detection accuracy and } \\
\text { time cost of the proposed detection system have } \\
\text { been detailed in the second last sentence of the } \\
\text { Abstract. }\end{array}$ \\
\hline 3 & $\begin{array}{l}\text { 1) The Introduction claims that DoS } \\
\text { attacks pose serious threats to IT } \\
\text { infrastructures, but does not present any } \\
\text { supporting evidence (e.g. from surveys, } \\
\text { threat reports etc) to illustrate the point. } \\
\text { 2) Similarly, it is asserted that toolkits are } \\
\text { readily available and anyone can use } \\
\text { them; it would be good to have some } \\
\text { evidence of (for example) the scale of } \\
\text { the actual problem that arises as a result. }\end{array}$ & $\begin{array}{l}\text { 1) To respond the reviewer's comment, [1] has } \\
\text { been cited as evidence to support the claim. } \\
\text { 2) To respond the reviewer's comment, [2] and } \\
\text { [3] have cited as evidence to support the } \\
\text { claim. }\end{array}$ \\
\hline 4 & $\begin{array}{l}\text { The later part of the Introduction indication } \\
\text { that the observed accuracy of the system is } \\
\text { "competitive to the previous systems", and } \\
\text { it may be worth indicating the associated } \\
\text { percentages for them as well at this point. }\end{array}$ & $\begin{array}{l}\text { The associated percentages of our proposed } \\
\text { system over the previous works have been } \\
\text { listed in detail. Please see the second and third } \\
\text { last sentence in the second last paragraph of the } \\
\text { Introduction on page } 2 \text {. }\end{array}$ \\
\hline 5 & $\begin{array}{l}\text { Section II presents a reasonable literature } \\
\text { review in terms of identifying related works } \\
\text { within the scope outlined at the start of the } \\
\text { section. However, one aspect that feels }\end{array}$ & $\begin{array}{l}\text { The results observed from the prior systems } \\
\text { have been reported in Section II as suggested. }\end{array}$ \\
\hline
\end{tabular}




\begin{tabular}{|c|c|c|}
\hline & $\begin{array}{l}\text { lacking is an indication of the } \\
\text { results/effectiveness observed from the prior } \\
\text { systems that are mentioned here. }\end{array}$ & \\
\hline 6 & $\begin{array}{l}\text { Section III.A presents good discussion of } \\
\text { the general mechanisms and the motivations } \\
\text { behind them. }\end{array}$ & We thank the reviewer's acknowledgement. \\
\hline 7 & $\begin{array}{l}\text { It is notable that, by the time of its mention } \\
\text { midway through the discussion on page } 6 \text {, } \\
\text { the choice of the KDD Cup } 99 \text { dataset has } \\
\text { not yet been fully explained. }\end{array}$ & $\begin{array}{l}\text { We thank the reviewer's comment. } \\
\text { However, the features in KDD Cup } 99 \text { data set } \\
\text { are just some examples. Raw features } \\
\text { mentioned in Section III-A5 could be features } \\
\text { other than those in KDD Cup } 99 \text { data set. }\end{array}$ \\
\hline 8 & $\begin{array}{l}\text { Section III as a whole serves to describe the } \\
\text { proposed approach in a significant amount } \\
\text { of detail. }\end{array}$ & We thank the reviewer's acknowledgement. \\
\hline 9 & $\begin{array}{l}\text { Section IV proceeds to present the rationale } \\
\text { for the KDD Cup } 99 \text { dataset, and } \\
\text { emphasizes the claim that the age of the } \\
\text { data is not a factor in this case. Nonetheless, } \\
\text { it would be useful to be able to include an } \\
\text { explicit statement regarding the } \\
\text { applicability of the proposed techniques to } \\
\text { use with more modern attacks and current } \\
\text { styles of network traffic. }\end{array}$ & $\begin{array}{l}\text { We thank the reviewer's comments and respond } \\
\text { the comments as follows. } \\
\text { First of all, new attacks will mostly likely bear } \\
\text { deviate patterns/behaviours than normal traffic. } \\
\text { So, modern attacks can remain being found } \\
\text { deviating from the current styles of network } \\
\text { traffic. } \\
\text { Furthermore, our approach is to model normal } \\
\text { network traffic. As long as the model is trained } \\
\text { and updated with the up-to-date network traffic, } \\
\text { it is capable of current network. } \\
\text { Finally, a new up-to-date data set, namely ISCX } \\
2012 \text { IDS Evaluation Dataset, has been used to } \\
\text { evaluate our proposed detection system. }\end{array}$ \\
\hline 10 & $\begin{array}{l}\text { Tables II and III do not appear to be very } \\
\text { useful or informative as currently presented. } \\
\text { 1) In the case of Table II, the information } \\
\text { could be stated without needing a table. } \\
\text { 2) For Table III, it is unclear from the table } \\
\text { why UDP and ICMP have multiple PC } \\
\text { values. }\end{array}$ & $\begin{array}{l}\text { 1) The original Table II has been removed } \\
\text { from the manuscript as suggested. The } \\
\text { numbers of principal components for the } \\
\text { respective network traffic are now specified } \\
\text { in the text. Please see the last paragraph of } \\
\text { Section IV-B1 on page } 10 \text {. } \\
\text { 2) We thank the reviewer's comment. The } \\
\text { reason why UDP and ICMP have multiple } \\
\text { PC values has been discussed in the last } \\
\text { paragraph of Section IV-B1. Since the best } \\
\text { performance may be achieved around the } \\
\text { numbers that we pick according to } \\
\text { accumulative variance plots, we should test } \\
\text { the numbers before and after the picked } \\
\text { ones. Thus, we have chosen two sets of }\end{array}$ \\
\hline
\end{tabular}




\begin{tabular}{|l|l|l|}
\hline 11 & $\begin{array}{l}\text { three different values for UDP and ICMP } \\
\text { respectively. In addition, the original Table } \\
\text { III, which is now Table I, has been revised } \\
\text { to enhance its readability. }\end{array}$ \\
\hline 12 & $\begin{array}{l}\text { The paper as a whole could benefit from } \\
\text { enhance readability. }\end{array}$ & $\begin{array}{l}\text { We have done our best to eradicate all language } \\
\text { (minor) language issues (e.g. two examples } \\
\text { from p12- "it is worth notice" should be "it } \\
\text { is worth noticing"; "in two folds" should be } \\
\text { "in two ways"). }\end{array}$ \\
\hline 13 & $\begin{array}{l}\text { The overall results to date appear positive in } \\
\text { terms of both accuracy and computational } \\
\text { complexity. It would be worth extending the } \\
\text { conclusion to discuss how the authors plan } \\
\text { to take things forward from this basis. }\end{array}$ & $\begin{array}{l}\text { Figure } 7 \text { has been enlarged as suggested. } \\
\text { future research focus, has been included in the } \\
\text { page 13 for details. }\end{array}$ \\
\hline
\end{tabular}


Responses to Reviewer 2's comments

\begin{tabular}{|l|l|l|}
\hline No. & \multicolumn{1}{|c|}{ Comments } & \multicolumn{1}{|c|}{ Revisions } \\
\hline 1 & $\begin{array}{l}\text { This paper proposed an innovative } \\
\text { research about original network intrusion } \\
\text { detection problem into a computer vision } \\
\text { task. A new scheme transforms the } \\
\text { network traffic records into computer } \\
\text { vision tasks. Multivariate Correlation } \\
\text { Analysis approaches are proposed to } \\
\text { improve the computing accuracy and } \\
\text { accelerate. In the data pre-treatment } \\
\text { partial, principal component analysis } \\
\text { algorithm is introduced to reduce the data } \\
\text { dimension. In the network traffic attack } \\
\text { detection partial, Earth Mover's Distance } \\
\text { model is employed to calculate the } \\
\text { similarity between observed traffic record } \\
\text { and normal profiles. This new method is } \\
\text { able to distinguish known and unknown } \\
\text { DoS attack from legitimate network } \\
\text { traffic. }\end{array}$ \\
\hline
\end{tabular}


Responses to Reviewer 3's comments

\begin{tabular}{|l|l|l|}
\hline No. & \multicolumn{1}{|c|}{ Comments } & \multicolumn{1}{|c|}{ Revisions } \\
\hline 1 & $\begin{array}{l}\text { The paper is well written and easy to } \\
\text { read. The structure and the analysis of the } \\
\text { proposed image based DoS detection }\end{array}$ & $\begin{array}{l}\text { We sincerely thank the reviewer's } \\
\text { acknowledgement. }\end{array}$ \\
$\begin{array}{l}\text { system are well presented. Performance } \\
\text { of the proposed system exceeds or } \\
\text { matches those of the existing state of the } \\
\text { art detection systems. }\end{array}$ & \\
\hline
\end{tabular}


Responses to Reviewer 4's comments

\begin{tabular}{|c|c|c|}
\hline No. & Comments & Revisions \\
\hline 1 & $\begin{array}{l}\text { This paper proposes a new method to } \\
\text { detect Internet distributed denial of } \\
\text { service attacks from a computer vision } \\
\text { perspective. This paper makes significant } \\
\text { contribution to the field. However, there } \\
\text { are many details need more investigation. } \\
\text { The methodology is sound; I do } \\
\text { appreciate its merits. However, the } \\
\text { justification of using such methodology } \\
\text { should be given in details. }\end{array}$ & $\begin{array}{l}\text { We thank the reviewer's comment. } \\
\text { Justification regarding the proposed methodology } \\
\text { has been presented in Sections I (the fifth } \\
\text { paragraph on page 2) and III-A4 (the first } \\
\text { paragraph on page 5). }\end{array}$ \\
\hline 2 & $\begin{array}{l}\text { The key weakness of this paper is the } \\
\text { evaluation method and dataset. } \\
\text { 1) There have been many types of } \\
\text { distributed denial of service attacks. } \\
\text { Therefore, different methods and } \\
\text { datasets should be used to evaluate } \\
\text { the effectiveness of the proposed } \\
\text { system. The authors should give } \\
\text { specific methods and datasets, and } \\
\text { give justification of using such } \\
\text { methods and datasets. } \\
\text { 2) In the paper, the dataset used, is not } \\
\text { convincing, as it is a very old dataset, } \\
\text { which cannot reflect the real situation } \\
\text { of the current Internet. Newer } \\
\text { datasets should be used. }\end{array}$ & $\begin{array}{l}\text { We thank the reviewer's comment. } \\
\text { 1) As suggested, justification for choosing KDD } \\
\text { Cup } 99 \text { data set and ISCX 2012 IDS } \\
\text { evaluation data set has been given in the } \\
\text { second and fourth paragraph of Section IV on } \\
\text { pages } 9 \text { and } 10 \text { respectively, and justification } \\
\text { for the chosen evaluation method has been } \\
\text { given in the second paragraph of Section IV-B } \\
\text { on page } 10 \text {. } \\
\text { 2) As suggested, a new data set, namely ISCX } \\
\text { 2012 IDS Evaluation Dataset, has been used to } \\
\text { evaluate our proposed detection system. Please } \\
\text { see Section IV for details. }\end{array}$ \\
\hline 3 & $\begin{array}{l}\text { The thresholds selected for attack } \\
\text { detection is arbitrary. This method is not } \\
\text { adaptive. There have been many other } \\
\text { adaptive methods, such as CUSUM (and } \\
\text { the authors should cite this paper). The } \\
\text { proposed system does not show } \\
\text { advantages against the previous methods. }\end{array}$ & $\begin{array}{l}\text { We thank the reviewer's comment. } \\
\text { 1) The threshold is determined according to the } \\
\text { distribution theory. For each traffic model, } \\
\text { there are always five candidate thresholds, } \\
\text { namely } 1 \sigma, 1.5 \sigma, 2 \sigma, 2.5 \sigma \text { and } 3 \sigma \text {, for } \\
\text { choosing. } \sigma \text { is the standard deviation of the } \\
\text { distribution of Earth Mover's Distance. We } \\
\text { choose the final threshold for a traffic model } \\
\text { according to its empirical performance in } \\
\text { testing. } \\
\text { 2) CUSUM has been cited. Please see reference } \\
\text { [22]. } \\
\text { 3) Incremental (adaptive) learning version of our } \\
\text { detection system has been introduced in the } \\
\text { last paragraph of Section III-C } 2 \text { on page } 9 \text {. }\end{array}$ \\
\hline
\end{tabular}


Prof. Albert Y. Zomaya

Editor-in-Chief

IEEE Transactions on Computers

4 November, 2014

Dear Prof. Zomaya:

I am pleased to submit an original research article entitled "Detection of Denial-of-Service Attacks Based on Computer Vision Techniques" by Zhiyuan Tan, Aruna Jamdagni, Xiangjian He, Priyadarsi Nanda, Ren Ping Liu and Jiankun Hu for consideration for publication in the IEEE Transactions on Computers.

In this submission, we propose a new system based on computer vision techniques for detecting DoS attacks. It differs from our preliminary study published in IEEE Transactions on Parallel and Distributed Systems (The paper was entitled "A System for Denial-of-Service Attack Detection Based on Multivariate Correlation Analysis") as follows.

This submission innovatively suggests reformulating the original network intrusion detection problem into a computer vision task (i.e., object shape recognition). The reformulation is motivated by the commonalities shared between network intrusion detection problem and object shape recognition tasks.

In addition, we propose a scheme to facilitate a rationale data transformation, which helps generate the image-like representations for the respective network traffic records. This enables the adaptation of the techniques, which were designed for computer vision tasks only, to network intrusion detection mission. In this new scheme, to improve the accuracy and to accelerate the computation of our previously proposed Multivariate Correlation Analysis approaches, Principal Component Analysis is employed to reduce the dimensionality (noise) of data. Furthermore, inbound network traffic records are converted into two-dimensional images before detection is conducted. The proposed data transformation scheme creates a paradigm for future studies of the fusion between network intrusion detection and computer vision.

Moreover, we employ the Earth Mover's Distance (which is a robust distance metric supporting partial matching and has been widely used in computer vision tasks) in our proposed attack detection system. The Earth Mover's Distance is used to evaluate the similarity between the observed network traffic records and the respective normal profiles from the perspective of computer vision. Thus, we can determine whether the observed network traffic records are attacks or legitimate one according the level of visual similarity. To the best of our knowledge, it is the first time that the Earth Mover's Distance has ever been applied in field of network DoS attack detection.

We believe that this manuscript is appropriate for publication by the IEEE Transactions on Computers. It proposes a new system to enhance the network security, and creates a paradigm for future studies of network intrusion detection based on computer vision techniques. This manuscript has not been published and is not under consideration for publication elsewhere. We have no conflicts of interest to disclose. Thank you for your consideration!

Sincerely,

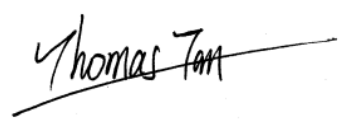

\section{Dr Zhiyuan Tan}

Services, Cybersecurity and Safety Research Group

Faculty of Electrical Engineering, Mathematics and Computer Science

University of Twente,

P.O. Box 217 7500AE Enschede

The Netherlands

Office: Zilverling 4037

Office Ph.: +31 534893455

E-mail: z.tan@utwente.nl 


\begin{abstract}
Interconnected systems, such as Web servers, database servers, cloud computing servers and so on, are now under threads from network attackers. As one of most common and aggressive means, denial-of-service (DoS) attacks cause serious impact on these computing systems. In this paper, we present a DoS attack detection system that uses multivariate correlation analysis (MCA) for accurate network traffic characterization by extracting the geometrical correlations between network traffic features. Our MCA-based DoS attack detection system employs the principle of anomaly based detection in attack recognition. This makes our solution capable of detecting known and unknown DoS attacks effectively by learning the patterns of legitimate network traffic only. Furthermore, a triangle-area-based technique is proposed to enhance and to speed up the process of MCA. The effectiveness of our proposed detection system is evaluated using KDD Cup 99 data set, and the influences of both non-normalized data and normalized data on the performance of the proposed detection system are examined. The results show that our system outperforms two other previously developed state-of-the-art approaches in terms of detection accuracy.
\end{abstract}

Index Terms-Denial-of-service attack, network traffic characterization, multivariate correlations, triangle area

\section{INTRODUCTION}

$\mathrm{D}$ ENIAL-OF-SERVICE (DoS) attacks are one type of aggressive and menacing intrusive behavior to online servers. DoS attacks severely degrade the availability of a victim, which can be a host, a router, or an entire network. They impose intensive computation tasks to the victim by exploiting its system vulnerability or flooding it with huge amount of useless packets. The victim can be forced out of service from a few minutes to even several days. This causes serious damages to the services running on the victim. Therefore, effective detection of DoS attacks is essential to the protection of online services. Work on DoS attack detection mainly focuses on the development of network-based detection mechanisms. Detection systems based on these mechanisms monitor traffic transmitting over the protected networks. These mechanisms release the

- Z. Tan is with the Centre for Innovation in IT Services and Applications (iNEXT), School of Computing and Communications, Faculty of Engineering and IT, University of Technology, Sydney, PO Box 123, Broadway, New South Wales 2007, Australia. E-mail: Zhiyuan.Tan@uts.edu.au.

- A. Jamdagni is with the School of Computing and Mathematics, University ofWestern Sydney, Parramatta, Australia.

E-mail:a.jamdagni@uws.edu.au.

- X. He and P. Nanda are with the Centre for Innovation in IT Services and Applications (iNEXT), School of Computing and Communications, University of Technology, Sydney, PO Box 123, Broadway, New South Wales 2007, Australia.

E-mail: \{Xiangjian.He, Priyadarsi.Nanda\}@uts.edu.au.

- R.P. Liu is with CSIRO ICT Centre, PO Box 76, Epping, New South Wales 1710, Australia. E-mail: ren.liu@csiro.au.

Manuscript received 16 Sept. 2012; revised 6 Apr. 2013; accepted 3 May 2013; published online 21 May 2013.

Recommended for acceptance by X. Li, P. McDaniel, R. Poovendran, and G. Wang.

For information on obtaining reprints of this article, please send e-mail to: tpds@computer.org, and reference IEEECS Log Number TPDSSI-2012-09-0881.

Digital Object Identifier no. 10.1109/TPDS.2013.146. protected online servers from monitoring attacks and ensure that the servers can dedicate themselves to provide quality services with minimum delay in response. Moreover, network-based detection systems are loosely coupled with operating systems running on the host machines which they are protecting. As a result, the configurations of network-based detection systems are less complicated than that of host-based detection systems.

Generally, network-based detection systems can be classified into two main categories, namely, misuse-based detection systems [1] and anomaly based detection systems [2]. Misuse-based detection systems detect attacks by monitoring network activities and looking for matches with the existing attack signatures. In spite of having high detection rates to known attacks and low false-positive rates, misuse-based detection systems are easily evaded by any new attacks and even variants of the existing attacks. Furthermore, it is a complicated and labor intensive task to keep signature database updated because signature generation is a manual process and heavily involves network security expertise.

Research community, therefore, started to explore a way to achieve novelty-tolerant detection systems and developed a more advanced concept, namely, anomaly based detection. Owing to the principle of detection, which monitors and flags any network activities presenting significant deviation from legitimate traffic profiles as suspicious objects, anomaly based detection techniques show more promising in detecting zero-day intrusions that exploit previous unknown system vulnerabilities [3]. Moreover, it is not constrained by the expertise in network security, due to the fact that the profiles of legitimate behaviors are developed based on techniques, such as data mining [4], [5], machine learning [6], [7], and statistical analysis [8], [9]. However, these proposed systems commonly suffer from high false-positive rates because 


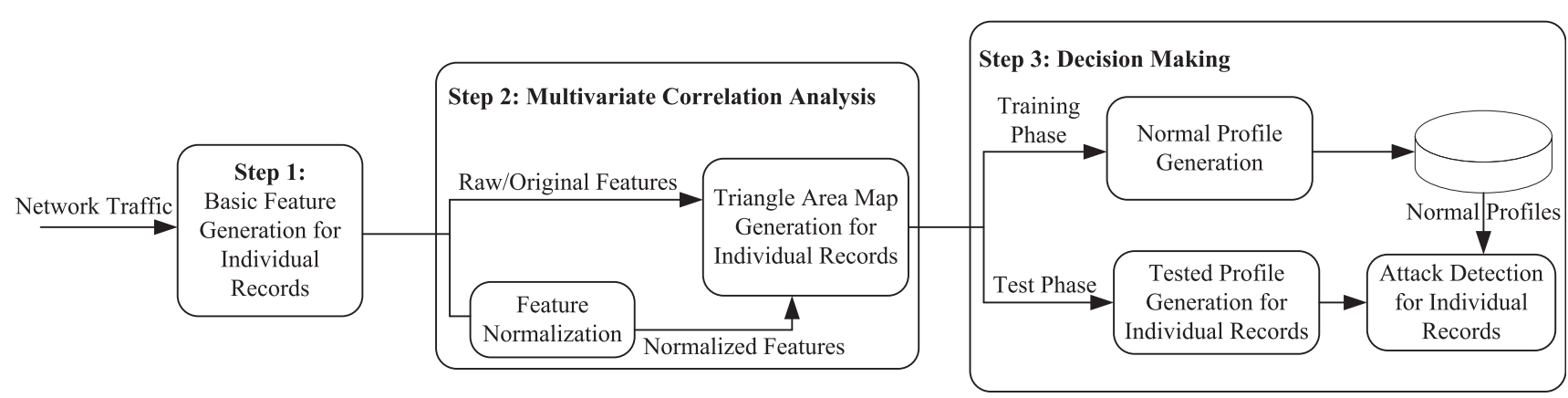

Fig. 1. Framework of the proposed denial-of-service attack detection system.

the correlations between features/attributes are intrinsically neglected [10] or the techniques do not manage to fully exploit these correlations.

Recent studies have focused on feature correlation analysis. Yu et al. [11] proposed an algorithm to discriminate DDoS attacks from flash crowds by analyzing the flow correlation coefficient among suspicious flows. A covariance matrix-based approach was designed in [12] to mine the multivariate correlation for sequential samples. Although the approach improves detection accuracy, it is vulnerable to attacks that linearly change all monitored features. In addition, this approach can only label an entire group of observed samples as legitimate or attack traffic but not the individuals in the group. To deal with the above problems, an approach based on triangle area was presented in [13] to generate better discriminative features. However, this approach has dependence on prior knowledge of malicious behaviors. More recently, Jamdagni et al. [14] developed a refined geometrical structure-based analysis technique, where Mahalanobis distance (MD) was used to extract the correlations between the selected packet payload features. This approach also successfully avoids the above problems, but it works with network packet payloads. In [15], Tan et al. proposed a more sophisticated nonpayload-based DoS detection approach using multivariate correlation analysis (MCA). Following this emerging idea, we present a new MCA-based detection system to protect online services against DoS attacks in this paper, which is built upon our previous work in [16]. In addition to the work shown in [16], we present the following contributions in this paper. First, we develop a complete framework for our proposed DoS attack detection system in Section 2.1. Second, we propose an algorithm for normal profile generation and an algorithm for attack detection in Sections 4.1 and 4.3, respectively. Third, we proceed a detailed and complete mathematical analysis of the proposed system and investigate further on time cost in Section 6. As resources of interconnected systems (such as Web servers, database servers, cloud computing servers, etc.) are located in service providers' local area networks that are commonly constructed using the same or alike network underlying infrastructure and are compliant with the underlying network model, our proposed detection system can provide effective protection to all of these systems by considering their commonality.

The DoS attack detection system presented in this paper employs the principles of MCA and anomaly based detection. They equip our detection system with capabilities of accurate characterization for traffic behaviors and detection of known and unknown attacks, respectively. A triangle area technique is developed to enhance and to speed up the process of MCA. A statistical normalization technique is used to eliminate the bias from the raw data. Our proposed DoS detection system is evaluated using KDD Cup 99 data set [17] and outperforms the state-of-theart systems shown in [13] and [15].

The remainder of this paper is organized as follows: We give the overview of the system architecture in Section 2. Section 3 presents a novel MCA technique. Section 4 describes our MCA-based detection mechanism. Section 5 evaluates the performance of our proposed detection system using KDD Cup 99 data set. Section 6 shows the systematic analysis on the computational complexity and the time cost of the proposed system. Finally, conclusions are drawn and future work is given in Section 7.

\section{System ARChitecture}

The overview of our proposed DoS attack detection system architecture is given in this section, where the system framework and the sample-by-sample detection mechanism are discussed.

\subsection{Framework}

The whole detection process consists of three major steps as shown in Fig. 1. The sample-by-sample detection mechanism is involved in the whole detection phase (i.e., Steps 1, 2, and 3) and is detailed in Section 2.2.

In Step 1, basic features are generated from ingress network traffic to the internal network where protected servers reside in and are used to form traffic records for a well-defined time interval. Monitoring and analyzing at the destination network reduce the overhead of detecting malicious activities by concentrating only on relevant inbound traffic. This also enables our detector to provide protection which is the best fit for the targeted internal network because legitimate traffic profiles used by the detectors are developed for a smaller number of network services. The detailed process can be found in [17].

Step 2 is multivariate correlation analysis, in which the "triangle area map generation" module is applied to extract the correlations between two distinct features within each traffic record coming from the first step or the traffic record normalized by the "feature normalization" module in this step (Step 2). The occurrence of network intrusions cause changes to these correlations so that the changes can be 
used as indicators to identify the intrusive activities. All the extracted correlations, namely, triangle areas stored in triangle area maps (TAMs), are then used to replace the original basic features or the normalized features to represent the traffic records. This provides higher discriminative information to differentiate between legitimate and illegitimate traffic records. Our MCA method and the feature normalization technique are explained in Sections 3 and 5.2 , respectively.

In Step 3, the anomaly based detection mechanism [3] is adopted in decision making. It facilitates the detection of any DoS attacks without requiring any attack relevant knowledge. Furthermore, the labor-intensive attack analysis and the frequent update of the attack signature database in the case of misuse-based detection are avoided. Meanwhile, the mechanism enhances the robustness of the proposed detectors and makes them harder to be evaded because attackers need to generate attacks that match the normal traffic profiles built by a specific detection algorithm. This, however, is a labor-intensive task and requires expertise in the targeted detection algorithm. Specifically, two phases (i.e., the "training phase" and the "test phase") are involved in decision making. The "normal profile generation" module is operated in the "training phase" to generate profiles for various types of legitimate traffic records, and the generated normal profiles are stored in a database. The "tested profile generation" module is used in the "test phase" to build profiles for individual observed traffic records. Then, the tested profiles are handed over to the "attack detection" module, which compares the individual tested profiles with the respective stored normal profiles. A threshold-based classifier is employed in the "attack detection" module to distinguish DoS attacks from legitimate traffic. The detailed algorithm is given in Section 4 .

\subsection{Sample-by-Sample Detection}

Jin et al. [12] systematically proved that the group-based detection mechanism maintained a higher probability in classifying a group of sequential network traffic samples than the sample-by-sample detection mechanism. Whereas the proof was based on an assumption that the samples in a tested group were all from the same distribution (class). This restricts the applications of the group-based detection to limited scenarios, because attacks occur unpredictably in general and it is difficult to obtain a group of sequential samples only from the same distribution.

To remove this restriction, our system in this paper investigates traffic samples individually. This offers benefits that are not found in the group-based detection mechanism. For example, 1) attacks can be detected in a prompt manner in comparison with the group-based detection mechanism, 2) intrusive traffic samples can be labeled individually, and 3) the probability of correctly classifying a sample into its population is higher than the one achieved using the group-based detection mechanism in a general network scenario. To better understand the merits, we illustrate them through a mathematical example given in [12], which assumes traffic samples are independent and identically distributed [12], [18], [19], and legitimate traffic and illegitimate traffic follow normal distributions
$X_{1} \sim N\left(\mu_{1}, \sigma_{1}^{2}\right)$ and $X_{2} \sim N\left(\mu_{2}, \sigma_{2}^{2}\right)$, respectively. The two distributions are described statistically using the probability density functions $f\left(x ; \mu_{1}, \sigma_{1}^{2}\right)=\left(1 /\left(\sigma_{1} \sqrt{2 \pi}\right)\right) e^{-\left(x-\mu_{1}\right)^{2} / 2 \sigma_{1}^{2}}$ and $f\left(x ; \mu_{2}, \sigma_{2}^{2}\right)=\left(1 /\left(\sigma_{2} \sqrt{2 \pi}\right)\right) e^{-\left(x-\mu_{2}\right)^{2} / 2 \sigma_{2}^{2}}$, respectively, where $x \in(-\infty,+\infty)$. In this task, the sample-by-sample labeling and the group-based labeling are used to identify the correct distribution for the individuals from a group of $k$ independent samples $\left\{x_{1}, x_{2}, \ldots, x_{k}\right\}$.

In [12], on one hand, Jin et al. defined the probabilities of correctly classifying a sample into its distribution using the sample-by-sample labeling as the cumulative distribution functions shown in (1) and (2), respectively,

$$
\left\{\begin{array}{l}
P_{1}=\int_{-\infty}^{\bar{\mu}} \frac{1}{\sigma_{1} \sqrt{2 \pi}} e^{-\left(x-\mu_{1}\right)^{2} / 2 \sigma_{1}^{2}} d x \\
P_{2}=\int_{\bar{\mu}}^{+\infty} \frac{1}{\sigma_{2} \sqrt{2 \pi}} e^{-\left(x-\mu_{2}\right)^{2} / 2 \sigma_{2}^{2}} d x
\end{array}\right.
$$

where $\bar{\mu}=\mu_{1} \times \frac{\sigma_{2}}{\sigma_{1}+\sigma_{2}}+\mu_{2} \times \frac{\sigma_{1}}{\sigma_{1}+\sigma_{2}}$ is the threshold value for classifying a sample into one of the two distributions $N\left(\mu_{1}, \sigma_{1}^{2}\right)$ and $N\left(\mu_{2}, \sigma_{2}^{2}\right) . P_{1}^{\prime}=1-P_{1}$ represents the probability that a sample coming from the distribution $N\left(\mu_{1}, \sigma_{1}^{2}\right)$ is not correctly classified into $X_{1} . P_{2}^{\prime}=1-P_{2}$ represents the probability that a sample coming from the distribution $N\left(\mu_{2}, \sigma_{2}^{2}\right)$ is not correctly classified into $X_{2}$. As proven in [12] that 1) $P_{1}=P_{2}=P$ and $P_{1}^{\prime}=P_{2}^{\prime}=1-P$, 2) the samples are independently distributive, and 3 ) the results of classification follow the binomial distribution, the probability of correctly labeling $j$ samples is defined as $\operatorname{Pr}(j)=C_{k}^{j} P^{j}(1-P)^{k-j}$ where $j=1,2, \ldots, k$. Thus, the probability of correctly classifying all $k$ samples is

$$
\operatorname{Pr}(k)=P^{k} \text {. }
$$

On the other hand, to classify the same group of independent samples $\left\{x_{1}, x_{2}, \ldots, x_{k}\right\}$ using the group-based labeling, a new random variable $z$, which is the mean of $k$ random samples from the distribution $N\left(\mu_{l}, \sigma_{l}^{2}\right)$, is defined as $z=\frac{1}{k} \sum_{t=1}^{k} x_{t}$, where $x_{t} \in X_{l}$ and $l=1,2$. Clearly, the new random variable $z$ follows the distribution $Z_{l} \sim N\left(\mu_{l}, \frac{1}{k} \sigma_{l}^{2}\right)$ in which $l=1,2$. The threshold value for classification is $\bar{u}=\mu_{1} \times \frac{\sigma_{2}}{\sigma_{1}+\sigma_{2}}+\mu_{2} \times \frac{\sigma_{1}}{\sigma_{1}+\sigma_{2}}$. Since the random variable $z$ is generated utilizing $k$ random samples $x_{t}$ from the distribution $N\left(\mu_{l}, \sigma_{l}^{2}\right)$, the detection precision rate of the $z$ correctly classified into the respective distribution $N\left(\mu_{1}, \sigma_{1}^{2}\right)$ or $N\left(\mu_{2}, \sigma_{2}^{2}\right)$ will thus be as given in (4) and (5), respectively.

$$
\left\{\begin{array}{l}
q_{1}=\int_{-\infty}^{\bar{u}}\left(1 /\left(\frac{1}{\sqrt{k}} \sigma_{1} \sqrt{2 \pi}\right)\right) e^{-\left(z-\mu_{1}\right)^{2} / \frac{2}{k} \sigma_{1}^{2}} d z, \\
q_{2}=\int_{\bar{u}}^{+\infty}\left(1 /\left(\frac{1}{\sqrt{k}} \sigma_{2} \sqrt{2 \pi}\right)\right) e^{-\left(z-\mu_{2}\right)^{2} / 2} \sigma_{k}^{2} \sigma_{2}^{2} d z .
\end{array}\right.
$$

As proven in [12], we have that $q_{1}=q_{2}, q_{1}^{\prime}=1-q_{1}$, and $q_{2}^{\prime}=1-q_{2}$.

The $z$ above represents a group of samples completely coming from the same distribution $N\left(\mu_{1}, \sigma_{1}^{2}\right)$ or $N\left(\mu_{2}, \sigma_{2}^{2}\right)$. However, in practice, samples may come from either distribution independently so that the probability of having a group of samples which come only from a single distribution $N\left(\mu_{1}, \sigma_{1}^{2}\right)$ or $N\left(\mu_{2}, \sigma_{2}^{2}\right)$ is $1 / 2^{k}$. Thus, the 
probability of correctly classifying all $k$ samples by using group-based labeling is

$$
\left\{\begin{array}{l}
k=1, Q(k)=q_{1}=q_{2}, \\
k>1, Q(k)=\frac{1}{2^{k}} q_{1}=\frac{1}{2^{k}} q_{2} .
\end{array}\right.
$$

Considering the same example given in [12, p. 2,188] where $k$ is set to 16 , the precision of the sample-by-sample labeling achieves $\operatorname{Pr}(16)=P^{16}=0.63^{16}=\mathbf{6 . 1 5 8 1 e - 0 4}$, and $q_{1}=q_{2}=0.90824$ when using group-based labeling. The precision of the group-based labeling achieving in the general network scenario is $Q(16)=\frac{1}{2^{16}} q_{1}=\frac{1}{2^{16}} \times 0.90824=$ 1.3859e-05. Clearly, the sample-by-sample labeling and the group-based labeling perform differently in detection precision. The relationship between the detection precisions of two detection mechanisms can be found by analyzing (3), (6), and (7). As shown in (8) and (9), when $k$ equals to 1 , the probability of correctly classifying all $k$ samples using the sample-by-sample labeling is same as the one using the group-based labeling. If $k$ is greater than 1 , both probabilities $\operatorname{Pr}(k)$ and $Q(k)$ decrease gradually, but the one of the groupbased labeling drops faster in comparison with that of the sample-by-sample labeling, i.e.,

$$
\left\{\begin{array}{l}
k=1, \operatorname{Pr}(k)=Q(k), \\
k>1, \operatorname{Pr}(k)>Q(k) .
\end{array}\right.
$$

Therefore, the sample-by-sample labeling can always achieve equal or better detection precision than the groupbased labeling.

\section{Multivariate Correlation Analysis}

DoS attack traffic behaves differently from the legitimate network traffic, and the behavior of network traffic is reflected by its statistical properties. To well describe these statistical properties, we present a novel MCA approach in this section. This MCA approach employs triangle area for extracting the correlative information between the features within an observed data object (i.e., a traffic record). The details are presented in the following.

Given an arbitrary data set $X=\left\{x_{1}, x_{2}, \ldots, x_{n}\right\}$, where $x_{i}=\left[f_{1}^{i} f_{2}^{i} \ldots f_{m}^{i}\right]^{T}, \quad(1 \leq i \leq n)$ represents the $i$ th $m$ dimensional traffic record. We apply the concept of triangle area to extract the geometrical correlation between the $j$ th and $k$ th features in the vector $x_{i}$. To obtain the triangle formed by the two features, data transformation is involved. The vector $x_{i}$ is first projected on the $(j, k)$ th $2 \mathrm{D}$ euclidean subspace as $y_{i, j, k}=\left[\varepsilon_{j} \varepsilon_{k}\right]^{T} x_{i}=\left[f_{j}^{i} f_{k}^{i}\right]^{T}$, $(1 \leq i \leq n, 1 \leq j \leq m, 1 \leq k \leq m, j \neq k)$. The vectors $\varepsilon_{j}=$ $\left[\begin{array}{llll}e_{j, 1} & e_{j, 2} & \ldots & e_{j, m}\end{array}\right]^{T}$ and $\varepsilon_{k}=\left[\begin{array}{llll}e_{k, 1} & e_{k, 2} & \ldots & e_{k, m}\end{array}\right]^{T}$ have elements with values of zero, except the $(j, j)$ th and $(k, k)$ th elements whose values are ones in $\varepsilon_{j}$ and $\varepsilon_{k}$, respectively. The $y_{i, j, k}$ can be interpreted as a $2 \mathrm{D}$ column vector, which can also be defined as a point on the Cartesian coordinate system in the $(j, k)$ th $2 \mathrm{D}$ euclidean subspace with coordinate $\left(f_{j}^{i}, f_{k}^{i}\right)$. Then, on the Cartesian coordinate system, a triangle $\Delta f_{j}^{i} O f_{k}^{i}$ formed by the origin and the projected points of the coordinate $\left(f_{j}^{i}, f_{k}^{i}\right)$ on the $j$-axis and $k$-axis is found. Its area $\operatorname{Tr}_{j, k}^{i}$ is defined as

$$
\operatorname{Tr}_{j, k}^{i}=\left(\left\|\left(f_{j}^{i}, 0\right)-(0,0)\right\| \times\left\|\left(0, f_{k}^{i}\right)-(0,0)\right\|\right) / 2,
$$

where $1 \leq i \leq n, 1 \leq j \leq m, 1 \leq k \leq m$, and $j \neq k$. To make a complete analysis, all possible permutations of any two distinct features in the vector $x_{i}$ are extracted and the corresponding triangle areas are computed. A TAM is constructed and all the triangle areas are arranged on the map with respect to their indexes. For example, the $\operatorname{Tr}_{j, k}^{i}$ is positioned on the $j$ th row and the $k$ th column of the map $T A M^{i}$, which has a size of $m \times m$. The values of the elements on the diagonal of the map are set to zeros $\left(\operatorname{Tr}_{j, k}^{i}=0\right.$, if $\left.j=k\right)$ because we only care about the correlation between each pair of distinct features. For the nondiagonal elements $\operatorname{Tr}_{j, k}^{i}$ and $\operatorname{Tr}_{k, j}^{i}$ where $j \neq k$, they indeed represent the areas of the same triangle. This infers that the values of $T r_{j, k}^{i}$ and $T r_{k, j}^{i}$ are actually equal. Hence, the $T A M^{i}$ is a symmetric matrix having elements of zero on the main diagonal.

When comparing two TAMs, we can imagine them as two images symmetric along their main diagonals. Any differences, identified on the upper triangles of the images, can be found on their lower triangles as well. Therefore, to perform a quick comparison of the two TAMs, we can choose to investigate either the upper triangles or the lower triangles of the TAMs only. This produces the same result as comparing using the entire TAMs (see Appendix 1, which can be found on the Computer Society Digital Library at http://doi.ieeecomputersociety.org/10.1109/ TPDS.2013.146). Therefore, the correlations residing in a traffic record (vector $x_{i}$ ) can be represented effectively and correctly by the upper triangle or the lower triangle of the respective $T A M^{i}$. For consistency, we consider the lower triangles of TAMs in the following sections. The lower triangle of the $T A M^{i}$ is converted into a new correlation vector $T A M_{\text {lower }}^{i}$ denoted as follows:

$$
\begin{aligned}
T A M_{\text {lower }}^{i}= & {\left[\begin{array}{llll}
\operatorname{Tr}_{2,1}^{i} & \operatorname{Tr}_{3,1}^{i} \ldots & \operatorname{Tr}_{m, 1}^{i} & \operatorname{Tr}_{3,2}^{i} \\
& \operatorname{Tr}_{4,2}^{i} \ldots r_{m, 2}^{i} & \ldots & T r_{m, m-1}^{i}
\end{array}\right]^{T} . }
\end{aligned}
$$

For the aforementioned data set $X$, its geometrical multivariate correlations can be represented by $X_{T A M_{\text {lower }}}=$ $\left\{T A M_{\text {lower }}^{1}, T A M_{\text {lower }}^{2}, \ldots, T A M_{\text {lower }}^{i}, \ldots, T A M_{\text {lower }}^{n}\right\}$.

When putting into practice, the computation of the $\operatorname{Tr}_{j, k}^{i}$ defined in (10) can be simplified because the value of the $\operatorname{Tr}_{j, k}^{i}$ is eventually equal to half of the multiplication of the absolute values of $f_{j}^{i}$ and $f_{k}^{i}$. Therefore, the transformation can be eliminated, and (10) can be replaced by $\operatorname{Tr}_{j, k}^{i}=$ $\left(\left|f_{j}^{i}\right| \times\left|f_{k}^{i}\right|\right) / 2$.

The above explanation shows that our MCA approach supplies with the following benefits to data analysis. First, it does not require the knowledge of historic traffic in performing analysis. Second, unlike the Covariance matrix approaches proposed in [12] which is vulnerable to linear change of all features, our proposed triangle-area-based MCA withstands the problem. Third, it provides characterization for individual network traffic records rather than model network traffic behavior of a group of network traffic records. This results in lower latency in decision making and enable sample-by-sample detection. Fourth, the correlations between distinct pairs of features are revealed through the geometrical structure analysis. Changes of 


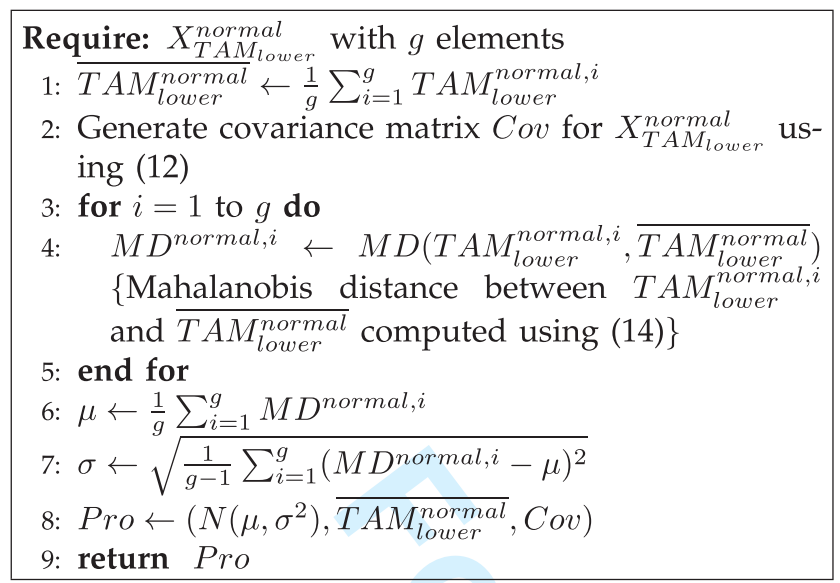

Fig. 2. Algorithm for normal profile generation based on triangle-areabased MCA.

these structures may occur when anomaly behaviors appear in the network. This provides an important signal to trigger an alert.

\section{Detection Mechanism}

In this section, we present a threshold-based anomaly detector, whose normal profiles are generated using purely legitimate network traffic records and utilized for future comparisons with new incoming investigated traffic records. The dissimilarity between a new incoming traffic record and the respective normal profile is examined by the proposed detector. If the dissimilarity is greater than a predetermined threshold, the traffic record is flagged as an attack. Otherwise, it is labeled as a legitimate traffic record. Clearly, normal profiles and thresholds have direct influence on the performance of a threshold-based detector. A low-quality normal profile causes an inaccurate characterization to legitimate network traffic. Thus, we first apply the proposed triangle-area-based MCA approach to analyze legitimate network traffic, and the generated TAMs are then used to supply quality features for normal profile generation.

\subsection{Normal Profile Generation}

Assume there is a set of $g$ legitimate training traffic records $X^{\text {normal }}=\left\{x_{1}^{\text {normal }}, x_{2}^{\text {normal }}, \ldots, x_{q}^{\text {normal }}\right\}$. The triangle-areabased MCA approach is applied to analyze the records. The generated lower triangles of the TAMs of the set of $g$ legitimate training traffic records are denoted by $X_{T A M_{\text {lower }}}^{\text {normal }}=\left\{T A M_{\text {lower }}^{\text {normal, } 1}, T A M_{\text {lower }}^{\text {normal }, 2}, \ldots, T A M_{\text {lower }}^{\text {normal, } g}\right\}$.

Mahalanobis distance is adopted to measure the dissimilarity between traffic records. This is because MD has been successfully and widely used in cluster analysis, classification and multivariate outlier detection techniques. Unlike euclidean distance and Manhattan distance, it evaluates distance between two multivariate data objects by taking the correlations between variables into account and removing the dependence on the scale of measurement during the calculation.

Fig. 2 presents the algorithm for normal profile generation, in which the normal profile Pro is built through the density estimation of the MDs between individual legitimate training traffic records $\left(T A M_{\text {lower }}^{\text {normal }, i}\right)$ and the expectation $\left(\overline{T A M_{\text {lower }}^{\text {normal }}}\right)$ of the $g$ legitimate training traffic records. The MD is computed using (14) and the covariance matrix $(C o v)$ involved in (14) can be obtained using the following equation:

$$
\begin{aligned}
& \operatorname{Cov}=\left[\begin{array}{c}
\sigma\left(\operatorname{Tr}_{2,1}^{\text {normal }}, \operatorname{Tr}_{2,1}^{\text {normal }}\right) \\
\sigma\left(\operatorname{Tr}_{3,1}^{\text {normal }}, \operatorname{Tr}_{2,1}^{\text {normal }}\right) \\
\vdots \\
\sigma\left(\operatorname{Tr}_{m, m-1}^{\text {normal }}, \operatorname{Tr}_{2,1}^{\text {normal }}\right)
\end{array}\right.
\end{aligned}
$$

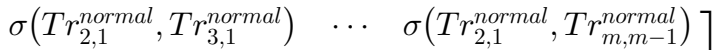

$$
\begin{aligned}
& \sigma\left(\operatorname{Tr}_{3,1}^{\text {normal }}, \operatorname{Tr}_{3,1}^{\text {normal }}\right) \quad \cdots \quad \sigma\left(\operatorname{Tr}_{3,1}^{\text {normal }}, \operatorname{Tr}_{m, m-1}^{\text {normal }}\right)
\end{aligned}
$$

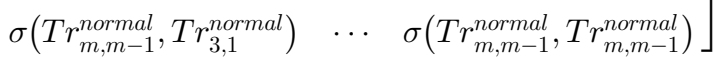

The covariance between two arbitrary elements in the lower triangle of a normal TAM is defined in (13).

$$
\begin{aligned}
\sigma\left(\operatorname{Tr}_{j, k}^{\text {normal }}, \operatorname{Tr}_{l, v}^{\text {normal }}\right)= & \frac{1}{g-1} \sum_{i=1}^{g}\left(\operatorname{Tr}_{j, k}^{\text {normal }, i}-\mu_{T r_{j, k}^{\text {normal }}}\right) \\
& \times\left(\operatorname{Tr}_{l, v}^{\text {normal }, i}-\mu_{T r_{l, v}^{\text {normal }}}\right) .
\end{aligned}
$$

Moreover, the mean of the $(j, k)$ th elements and the mean of the $(l, v)$ th elements of TAMs over $g$ legitimate training traffic records are defined as $\mu_{T r^{\text {normal }}}=\frac{1}{q} \sum_{i=1}^{g} \operatorname{Tr}_{j, k}^{\text {normal }, i}$ and $\mu_{T r_{l, v}^{\text {normal }}}=\frac{1}{g} \sum_{i=1}^{g} \operatorname{Tr}_{l, v}^{\text {normal }, i}$, respectively,

$$
\begin{aligned}
& M D^{\text {normal }, i}=
\end{aligned}
$$

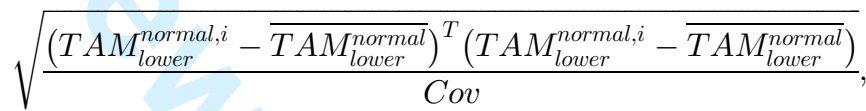

$$
\begin{aligned}
& M D^{\text {observed }}=
\end{aligned}
$$

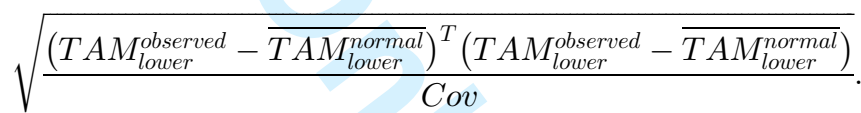

As shown in Fig. 2, the distribution of the MDs is described by two parameters, namely the mean $\mu$ and the standard deviation $\sigma$ of the MDs. Finally, the obtained distribution $N\left(\mu, \sigma^{2}\right)$ of the normal training traffic records, $\overline{T A M_{\text {lower }}^{\text {normal }}}$ and $C o v$ are stored in the normal profile Pro for attack detection.

\subsection{Threshold Selection}

The threshold given in (16) is used to differentiate attack traffic from the legitimate one

$$
\text { Threshold }=\mu+\sigma * \alpha .
$$

For a normal distribution, $\alpha$ is usually ranged from 1 to 3 . This means that detection decision can be made with a certain level of confidence varying from 68 to 99.7 percent in 


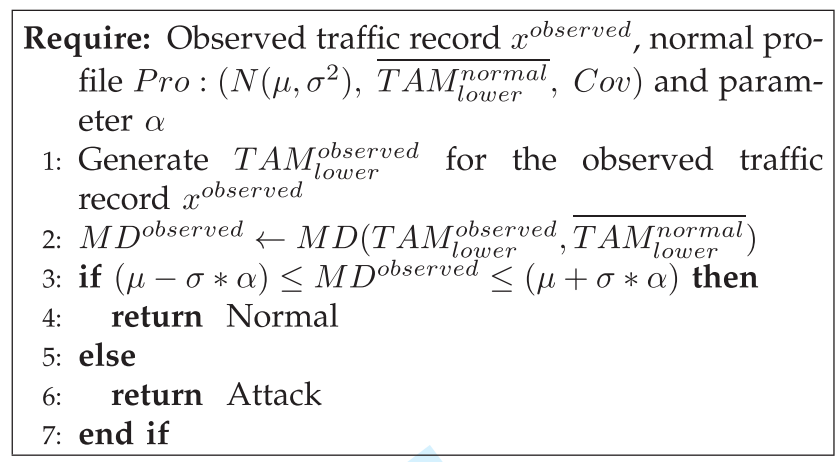

Fig. 3. Algorithm for attack detection based on Mahalanobis distance.

association with the selection of different values of $\alpha$. Thus, if the MD between an observed traffic record $x^{\text {observed }}$ and the respective normal profile is greater than the threshold, it will be considered as an attack. Attack detection is detailed in Section 4.3 .

\subsection{Attack Detection}

To detect DoS attacks, the lower triangle ( $\left.T A M_{\text {lower }}^{\text {observed }}\right)$ of the TAM of an observed record needs to be generated using the proposed triangle-area-based MCA approach. Then, the MD between the $T A M_{\text {lower }}^{\text {observed }}$ and the $T A M_{\text {lower }}^{\text {normal }}$ stored in the respective pregenerated normal profile Pro is computed using (15). The detailed detection algorithm is shown in Fig. 3.

\section{Evaluation of the MCA-Based Dos Attack Detection System}

The evaluation of our proposed DoS attack detection system is conducted using KDD Cup 99 data set [17]. Despite the data set is criticised for redundant records that prevent algorithms from learning infrequent harmful records [21], it is the only publicly available labeled benchmark data set, and it has been widely used in the domain of intrusion detection research. Testing our approach on KDD Cup 99 data set contributes a convincing evaluation and makes the comparisons with other state-of-the-art techniques equitable. Additionally, our detection system innately withstands the negative impact introduced by the data set because its profiles are built purely based on legitimate network traffic. Thus, our system is not affected by the redundant records.

During the evaluation, the 10 percent labeled data of KDD Cup 99 data set is used, where three types of legitimate traffic (TCP, UDP, and ICMP traffic) and six different types of DoS attacks (Teardrop, Smurf, Pod, Neptune, Land and Back attacks) are available. All of these records are first filtered and then are further grouped into seven clusters according to their labels (see Table 9 in Appendix 4, which is available in the online supplemental material).

The overall evaluation process is detailed as follows: First, the proposed triangle-area-based MCA approach is assessed for its capability of network traffic characterization. Second, a tenfold cross-validation is conducted to evaluate the detection performance of the proposed MCAbased detection system, and the entire filtered data subset is used in this task. In the training phase, we employ only the normal records. Normal profiles are built with respect

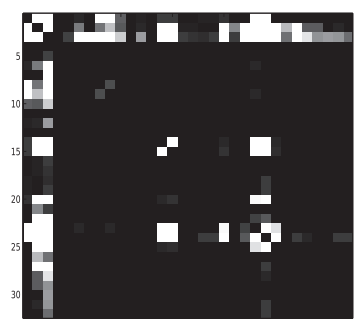

(a) Normal TCP record

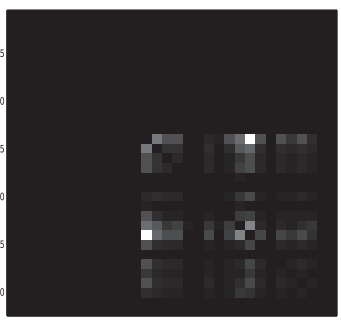

(c) Land attack record

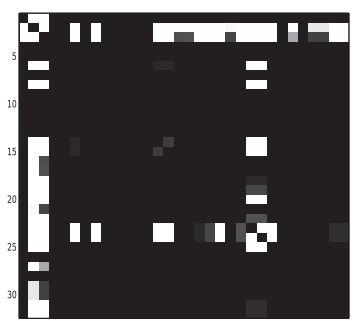

(b) Back attack record

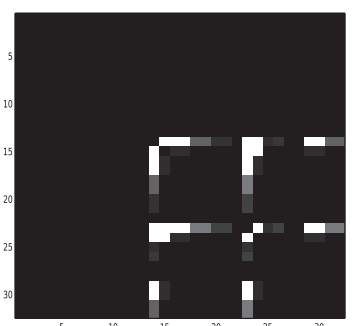

(d) Neptune attack record

Fig. 4. Images of TAMs of normal TCP traffic, Back, Land and Neptune attacks generated using original data.

to the different types of legitimate traffic using the algorithm presented in Fig. 2. The corresponding thresholds are determined according to (16) given the parameter $\alpha$ varying from 1 to 3 with an increment of 0.5 . During the test phase, both the Normal records and the attack records are taken into account. As given in Fig. 3, the observed samples are examined against the respective normal profiles which are built based on the legitimate traffic records carried using the same type of transport layer protocol. Third, four metrics, namely, true-negative rate (TNR), detection rate (DR), false-positive rate (FPR), and accuracy (i.e., the proportion of the overall samples which are classified correctly), are used to evaluate the proposed MCA-based detection system. To be a good candidate, our proposed detection system is required to achieve a high detection accuracy.

\subsection{Results and Analysis on Original Data}

\subsubsection{Network Traffic Characterization Using Triangle- Area-Based Multivariate Correlation Analysis}

In the evaluation, the TAMs of the different types of traffic records are generated using 32 continuous features. The images for the TAMs of Normal TCP record, Back attack record, Land attack record, and Neptune attack record are presented in Fig. 4. More results can be found in Appendix 2, which is available in the online supplemental material. The images demonstrate that TAM is a symmetric matrix, whose upper triangle and lower triangle are identical. The brightness of an element in an image represents its value in the corresponding TAM. The greater the value is, the brighter the element is. The images in Fig. 4 also demonstrate that our proposed MCA approach fulfils the anticipation of generating features for accurate network traffic characterization. 
TABLE 1

Average Detection Performance of the Proposed System on Original Data against Different Thresholds

\begin{tabular}{|l|l|l|l|l|l|}
\hline \multirow{2}{*}{$\begin{array}{l}\text { Type of } \\
\text { records }\end{array}$} & \multicolumn{5}{|c|}{ Threshold } \\
\cline { 2 - 6 } & $1 \sigma$ & $1.5 \sigma$ & $2 \sigma$ & $2.5 \sigma$ & $3 \sigma$ \\
\hline \hline Normal & $98.74 \%$ & $99.03 \%$ & $99.23 \%$ & $99.35 \%$ & $99.47 \%$ \\
\hline Teardrop & $71.50 \%$ & $63.92 \%$ & $57.93 \%$ & $52.81 \%$ & $48.45 \%$ \\
\hline Smurf & $100.00 \%$ & $100.00 \%$ & $100.00 \%$ & $100.00 \%$ & $100.00 \%$ \\
\hline Pod & $100.00 \%$ & $100.00 \%$ & $100.00 \%$ & $100.00 \%$ & $100.00 \%$ \\
\hline Neptune & $82.44 \%$ & $61.79 \%$ & $57.00 \%$ & $54.84 \%$ & $52.96 \%$ \\
\hline Land & $0.00 \%$ & $0.00 \%$ & $0.00 \%$ & $0.00 \%$ & $0.00 \%$ \\
\hline Back & $99.96 \%$ & $99.82 \%$ & $99.58 \%$ & $99.44 \%$ & $99.31 \%$ \\
\hline
\end{tabular}

\subsubsection{Tenfold Cross-Validation}

To evaluate the performance of our detection system along with the change of the threshold, the average TNRs for legitimate traffic and the average DRs for the individual types of DoS attacks are shown in Table 1.

Throughout the evaluation, our proposed detection system achieves encouraging performance in most of the cases except Land attack. The rate of correct classification of the Normal records rises from 98.74 to 99.47 percent along with the increase of the threshold. Meanwhile, the Smurf and Pod attack records are completely detected without being affected by the change of the threshold. Moreover, the system achieves nearly 100 percent DRs for the Back attacks in almost all cases. However, the detection system suffers serious degeneration in the cases of the Teardrop and Neptune attacks when the threshold is greater than $1.5 \sigma$. The DRs for these two attacks drop sharply to 48.45 and 52.96 percent, respectively, while the threshold is set to $3 \sigma$.

To have a better overview of the performance of our MCA-based detection system, the overall FPR and DR are highlighted in Table 2. The overall FPR and DR are computed over all traffic records regardless the types of attacks. When the threshold grows from $1 \sigma$ to $3 \sigma$, the FPR drops quickly from 1.26 to 0.53 percent. Correspondingly, the DR also drops from 95.11 to 86.98 percent while the threshold rises. It shows clearly in the table that a larger number of legitimate traffic records are covered by a greater threshold, and more DoS attack records are incorrectly accepted as legitimate traffic in the meantime.

\subsection{Problems with the Current System and Solution}

Although the detection system achieves a moderate overall detection performance in the above evaluation, we want to explore the causes of degradation in detecting the Land, Teardrop, and Neptune attacks.

Our analysis shows that the problems come from the data used in the evaluation, where the basic features in the non-normalized original data are in different scales. Therefore, even though our triangle-area-based MCA approach is promising in characterization and clearly reveals the patterns of the various types of traffic records, our detector is still ineffective in some of the attacks. For instance, the Land, Teardrop and Neptune attacks whose patterns are different than the patterns of the legitimate traffic. However, the level of the dissimilarity between these attacks and the respective normal profiles is close to that between the legitimate traffic and the respective normal profiles.
TABLE 2

Detection Rate and False-Positive Rate Achieved by the Proposed System on Original Data

\begin{tabular}{|l|l|l|l|l|l|}
\hline \multirow{2}{*}{} & \multicolumn{5}{|c|}{ Threshold } \\
\cline { 2 - 6 } & $1 \sigma$ & $1.5 \sigma$ & $2 \sigma$ & $2.5 \sigma$ & $3 \sigma$ \\
\hline \hline FPR & $1.26 \%$ & $0.97 \%$ & $0.77 \%$ & $0.65 \%$ & $0.53 \%$ \\
\hline DR & $95.11 \%$ & $89.44 \%$ & $88.11 \%$ & $87.51 \%$ & $86.98 \%$ \\
\hline Accuracy & $95.20 \%$ & $89.67 \%$ & $88.38 \%$ & $87.79 \%$ & $87.28 \%$ \\
\hline
\end{tabular}

Moreover, the changes appearing in some other more important features with much smaller values can hardly take effect in distinguishing the DoS attack traffic from the legitimate traffic, because the overall dissimilarity is dominated by the features with large values. Nevertheless, the non-normalized original data contains zero values in some of the features (both the important and the less important features), and they confuse our MCA and make many new generated features $\left(T r_{j, k}^{i}\right)$ equal to zeros. This vitally degrades the discriminative power of the new feature set $\left(T A M_{\text {lower }}^{i}\right)$, which is not supposed to happen.

Apparently, an appropriate data normalization technique should be employed to eliminate the bias. We adopt the statistical normalization technique [20] to this work. The statistical normalization takes both the mean scale of attribute values and their statistical distribution into account. It converts data derived from any normal distribution into standard normal distribution, in which 99.9 percent samples of the attribute are scaled into [-3, 3]. In addition, statistical normalization has been proven improving detection performance of distance-based classifiers and outperforming other normalization methods, such as mean range $[0,1]$, ordinal normalization and so on [20].

Considering the same arbitrary data set $X=\left\{x_{1}\right.$, $\left.x_{2}, \ldots, x_{n}\right\}$ given in Section 3, the statistical normalization is defined as follows: The normalized value of feature $f_{j}^{i}$ is given as $F_{j}^{i}=\left(f_{j}^{i}-\bar{f}_{j}\right) / \sigma_{f_{j}^{i}}$, where $\bar{f}_{j}=\frac{1}{n} \sum_{i=1}^{n} f_{j}^{i}$ is the mean of feature $f_{j}^{i}$, and

$$
\sigma_{f_{j}^{i}}=\sqrt{\frac{1}{n} \sum_{i=1}^{n}\left(f_{j}^{i}-\bar{f}_{j}\right)^{2}}
$$

is the standard deviation of feature $f_{j}^{i}$. The normalized feature vector $x_{i}$ is represented by $\left[F_{1}^{i} F_{2}^{i} \ldots F_{m}^{i}\right]^{T}$ in which $1 \leq i \leq n$. In the following evaluation, the data are normalized in a batch manner. However, real-time normalization can be achieved through the incremental learning [22] when our detection system is put online. The mean $f_{i}$ can be updated as $\bar{f}_{i}=\bar{f}_{i}+\frac{x_{n+1}-\bar{f}_{i}}{n+1}$.

\subsection{Results and Analysis on Normalized Data}

To verify our observation, a tenfold cross-validation is conducted as done in Section 5.1.2 on the data normalized using the aforementioned statistical normalization technique. The results are given in Section 5.3.1.

\subsubsection{Tenfold Cross-Validation}

The detection performance based on the normalized data is given in Table 3 . The results reveal that the data does have 
TABLE 3

Average Detection Performance of the Proposed System on Normalized Data against Different Thresholds

\begin{tabular}{|l|l|l|l|l|l|}
\hline \multirow{2}{*}{$\begin{array}{l}\text { Type of } \\
\text { records }\end{array}$} & \multicolumn{5}{|c|}{ Threshold } \\
\cline { 2 - 6 } & $1 \sigma$ & $1.5 \sigma$ & $2 \sigma$ & $2.5 \sigma$ & $3 \sigma$ \\
\hline \hline Normal & $97.36 \%$ & $97.97 \%$ & $98.32 \%$ & $98.56 \%$ & $98.75 \%$ \\
\hline Teardrop & $100.00 \%$ & $100.00 \%$ & $100.00 \%$ & $100.00 \%$ & $100.00 \%$ \\
\hline Smurf & $100.00 \%$ & $100.00 \%$ & $100.00 \%$ & $100.00 \%$ & $100.00 \%$ \\
\hline Pod & $100.00 \%$ & $100.00 \%$ & $100.00 \%$ & $100.00 \%$ & $100.00 \%$ \\
\hline Neptune & $100.00 \%$ & $100.00 \%$ & $100.00 \%$ & $100.00 \%$ & $100.00 \%$ \\
\hline Land & $100.00 \%$ & $100.00 \%$ & $100.00 \%$ & $100.00 \%$ & $100.00 \%$ \\
\hline Back & $99.32 \%$ & $98.96 \%$ & $94.09 \%$ & $93.79 \%$ & $93.56 \%$ \\
\hline
\end{tabular}

significant influence on our detection system, whose overall performance increases dramatically when taking the normalized data as the inputs. The Teardrop, Neptune, and Land attacks, which are mostly miss-classified in the previous evaluation, now can be completely classified correctly by the system along the increase of the threshold. Except the Back attacks, the other types of DoS attacks are detected completely regardless of the change of the threshold as well. Although the detection system claims only a 93.56 percent DR in detecting the Back attacks in the worst case, its DR rises stably and slowly to 99.32 percent when the a more rigorous threshold is chosen. The ineffectiveness of the statistical normalization technique on the Back attacks is caused by the fact that the non-normalized features of the Back attacks originally fall in similar scales as the ones of the legitimate traffic so that after data normalization there is no improvement on the detection of the Back attacks. In comparison with the TNR of our detection system achieved on the non-normalized Normal records, the one achieved on the normalized Normal records declines a bit to maximum 98.75 percent when the threshold is set to $3 \sigma$. However, it manages to remain in the reasonable range.

Then, similar to the previous evaluation, we show the overall FPR and DR in Table 4. The FPR shown in the table drops nearly 1 percent when the threshold increases from $1 \sigma$ to $2 \sigma$. Finally, it reaches to 1.25 percent while the threshold is staying at $3 \sigma$. The DR of the system varies from 100.00 to 99.96 percent. It is clearly seen that the proposed detection system achieves a better DR with the normalized data.

\subsubsection{Performance Comparisons}

To make complete comparisons, the ROC curves of the previous two evaluations are shown in Fig. 5. The relationship between DR and FPR is clearly revealed in the ROC curves. The DR increases when larger numbers of false positive are tolerated. In Fig. $5 a$, the ROC curve for analyzing the original data using our proposed detection system shows a rising trend. The curve climbs gradually from 86.98 to 89.44 percent DR, and finally reaches to 95.11 percent DR. Likewise, the ROC curve for analyzing the normalized data presents a resembling pattern but jumps dramatically from 99.97 to 99.99 percent DR after experiencing slow progress as shown in Fig. $5 b$. Then, the curve remains in a high level of DR around 100.00 percent. It is shown clearly in Fig. 5 that our detection system always enjoys higher detection rates
TABLE 4

Detection Rate and False-Positive Rate Achieved by the Proposed System on Normalized Data

\begin{tabular}{|l|l|l|l|l|l|}
\hline \multirow{2}{*}{} & \multicolumn{5}{|c|}{ Threshold } \\
\cline { 2 - 6 } & $1 \sigma$ & $1.5 \sigma$ & $2 \sigma$ & $2.5 \sigma$ & $3 \sigma$ \\
\hline \hline FPR & $2.64 \%$ & $2.03 \%$ & $1.68 \%$ & $1.44 \%$ & $1.25 \%$ \\
\hline DR & $100.00 \%$ & $99.99 \%$ & $99.97 \%$ & $99.97 \%$ & $99.96 \%$ \\
\hline Accuracy & $99.93 \%$ & $99.95 \%$ & $99.93 \%$ & $99.93 \%$ & $99.93 \%$ \\
\hline
\end{tabular}

while working with the normalized data than with the original data. The worst performance (99.96 percent DR and 1.25 percent FPR) of our system shown in Fig. $5 b$ is even much better the best performance ( 95.11 percent DR and 1.26 percent FPR) in term of detection rate shown in Fig. 5 a.

Last but not the least, two state-of-the-art detection approaches, namely, triangle area-based nearest neighbors approach [13] and euclidean distance map-based approach [15] are selected to compare with our proposed detection system. The best accuracies on detecting DoS attacks achieved by the various approaches and systems are given in Table 5. Although all approaches and systems highlighted in Table 5 have high accuracies on DoS attack detection, our proposed MCA-based detection system (95.20 percent for the original data and 99.95 percent for the normalized data) clearly outperforms the triangle areabased nearest neighbors approach (92.15 percent). In addition, our proposed detection system cooperating with normalized data (99.95 percent) shows a marginal advantage over the approach based on euclidean distance map (99.87 percent). Although this is a narrow lead, our detection system shows more promising especially when it is deployed on a production network with a throughput of $1 \mathrm{Gbps}$. Due to a significantly fewer number of false alarms generated per second, network administrators will be much less interrupted by the false information.

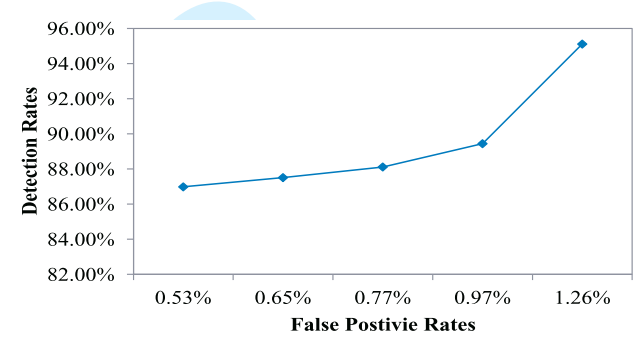

(a) ROC curve for analyzing original data

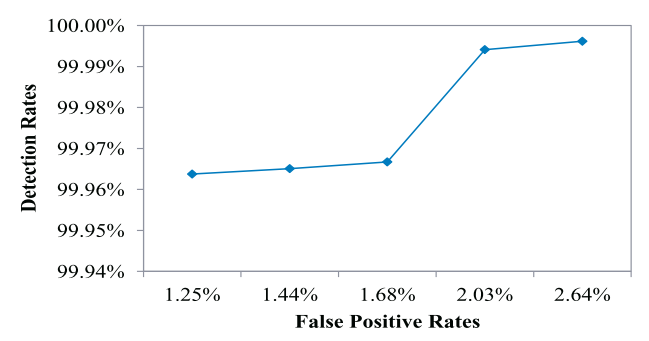

(b) ROC curve for analyzing normalized data

Fig. 5. ROC curves for the detection of DoS attacks. 


\section{Computational Complexity and Time Cost ANALYSIS}

In this section, we conduct an analysis on the computational complexity and the time cost of our proposed MCA-based detection system.

On one hand, as discussed in Section 3, triangle areas of all possible combinations of any two distinct features in a traffic record need to be computed when processing our proposed MCA. Since each traffic record has $m$ features (or dimensions), $\frac{m(m-1)}{2}$ triangle areas are generated and are used to construct a $T A M_{\text {lower }}^{i}$. Thus, the proposed MCA has a computational complexity of $O\left(\mathrm{~m}^{2}\right)$. On the other hand, as explained in Section 4.3, the MD between the observed

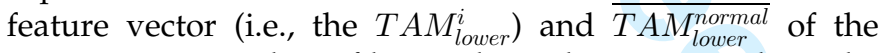
respective normal profile needs to be computed in the detection process of our proposed detection system to evaluate the level of the dissimilarity between them. Thus, this computation incurs a complexity of $O\left(M^{2}\right)$, in which $M=\frac{m(m-1)}{2}$ is the dimensions of $T A M_{\text {lower }}^{i} . O\left(M^{2}\right)$ can be written as $O\left(m^{4}\right)$. By taking the computational complexities of the proposed MCA and the detection process of our proposed detection system into account, the overall computational complexity of the proposed detection system is $O\left(m^{2}\right)+O\left(m^{4}\right)=O\left(m^{4}\right)$. However, $m$ is a fixed number which is 32 in our case, so that the overall computational complexity is indeed equal to $O(1)$.

Similarly, approach based on euclidean distance map [15] achieves the same computational complexities of $O\left(\mathrm{~m}^{2}\right)$ and $O\left(m^{4}\right)$ in data processing and attack detection, respectively. Moreover, the number of features $(m)$ in use is identical to that used in our proposed detection system as well. Thus, the overall computational complexity of the euclidean distance map-based approach is $O(1)$. For another state-of-the-art detection approach that we compared in the previous section, triangle area-based nearest neighbors approach [13] suffers a heavier overall computational complexity. In data processing and attack detection phases, the computational complexities are $O\left(m l^{2}\right)$ and $O\left(l^{2} n^{2}\right)$, respectively, where $m$ is the number of features (or dimensions) in a traffic record, $l$ is the number of clusters used in generating triangle areas and $n$ is the number of training samples. The overall complexity is $O\left(m l^{2}\right)+O\left(l^{2} n^{2}\right)=O\left(l^{2} n^{2}\right)$. In general, our proposed detection system can achieve equal or better computational

TABLE 6

Computational Complexities of Different State-of-the-Art Detection Approaches

\begin{tabular}{|c|c|c|}
\hline $\begin{array}{c}\text { The proposed } \\
\text { detection system }\end{array}$ & $\begin{array}{c}\text { Euclidean distance map } \\
\text { based approach [15] }\end{array}$ & $\begin{array}{c}\text { Triangle area based nearest } \\
\text { neighbors approach [13] }\end{array}$ \\
\hline \hline$O(1)$ & $O(1)$ & $O\left(l^{2} n^{2}\right)$ \\
\hline
\end{tabular}

complexity than the above two other approaches. Table 6 is provided to summarize the computational complexities of the above discussed approaches.

Moreover, time cost is discussed to show the contribution of our proposed MCA in terms of acceleration of data processing. Our proposed MCA can proceed approximately 23,092 traffic records per second. In contrast, the MCA based on euclidean distance map [15] can achieve approximately 12,044 traffic records per second, which is nearly less than half of that achieved by our proposed MCA. Due to the unavailability of the source code of triangle area-based nearest neighbors approach [13], we cannot provide comparison to it.

\section{Conclusion AND Future Work}

This paper has presented an MCA-based DoS attack detection system which is powered by the triangle-areabased MCA technique and the anomaly-based detection technique. The former technique extracts the geometrical correlations hidden in individual pairs of two distinct features within each network traffic record, and offers more accurate characterization for network traffic behaviors. The latter technique facilitates our system to be able to distinguish both known and unknown DoS attacks from legitimate network traffic.

Evaluation has been conducted using KDD Cup 99 data set to verify the effectiveness and performance of the proposed DoS attack detection system. The influence of original (non-normalized) and normalized data has been studied in the paper. The results have revealed that when working with non-normalized data, our detection system achieves maximum 95.20 percent detection accuracy although it does not work well in identifying Land, Neptune, and Teardrop attack records. The problem, however, can be solved by utilizing statistical normalization technique to eliminate the bias from the data. The results of evaluating with the normalized data have shown a more encouraging detection accuracy of 99.95 percent and nearly 100.00 percent DRs for the various DoS attacks. Besides, the comparison result has proven that our detection system outperforms two state-of-the-art approaches in terms of detection accuracy. Moreover, the computational complexity and the time cost of the proposed detection system have been analyzed and shown in Section 6. The proposed system achieves equal or better performance in comparison with the two state-of-the-art approaches.

To be part of the future work, we will further test our DoS attack detection system using real-world data and employ more sophisticated classification techniques to further alleviate the false-positive rate.

\section{ACKNOWLEDGMENTS}

Xiangiian He is the corresponding author. 


\section{1}

\section{References}

[1] V. Paxson, "Bro: A System for Detecting Network Intruders in Real-Time," Computer Networks, vol. 31, pp. 2435-2463, 1999.

[2] P. Garca-Teodoro, J. Daz-Verdejo, G. Maci-Fernndez, and E. Vzquez, "Anomaly-Based Network Intrusion Detection: Techniques, Systems and Challenges," Computers and Security, vol. 28, pp. 18-28, 2009.

[3] D.E. Denning, "An Intrusion-Detection Model," IEEE Trans. Software Eng., vol. TSE-13, no. 2, pp. 222-232, Feb. 1987.

[4] K. Lee, J. Kim, K.H. Kwon, Y. Han, and S. Kim, "DDoS Attack Detection Method Using Cluster Analysis," Expert Systems with Applications, vol. 34, no. 3, pp. 1659-1665, 2008.

[5] A. Tajbakhsh, M. Rahmati, and A. Mirzaei, "Intrusion Detection Using Fuzzy Association Rules," Applied Soft Computing, vol. 9, no. 2, pp. 462-469, 2009.

[6] J. Yu, H. Lee, M.-S. Kim, and D. Park, "Traffic Flooding Attack Detection with SNMP MIB Using SVM," Computer Comm., vol. 31, no. 17 , pp. 4212-4219, 2008.

[7] W. Hu, W. Hu, and S. Maybank, "AdaBoost-Based Algorithm for Network Intrusion Detection," IEEE Trans. Systems, Man, and Cybernetics Part B, vol. 38, no. 2, pp. 577-583, Apr. 2008.

[8] C. Yu, H. Kai, and K. Wei-Shinn, "Collaborative Detection of DDoS Attacks over Multiple Network Domains," IEEE Trans. Parallel and Distributed Systems, vol. 18, no. 12, pp. 1649-1662, Dec. 2007.

[9] G. Thatte, U. Mitra, and J. Heidemann, "Parametric Methods for Anomaly Detection in Aggregate Traffic," IEEE/ACM Trans. Networking, vol. 19, no. 2, pp. 512-525, Apr. 2011.

[10] S.T. Sarasamma, Q.A. Zhu, and J. Huff, "Hierarchical Kohonenen Net for Anomaly Detection in Network Security," IEEE Trans. Systems, Man, and Cybernetics, Part B: Cybernetics, vol. 35, no. 2, pp. 302-312, Apr. 2005.

[11] S. Yu, W. Zhou, W. Jia, S. Guo, Y. Xiang, and F. Tang, "Discriminating DDoS Attacks from Flash Crowds Using Flow Correlation Coefficient," IEEE Trans. Parallel and Distributed Systems, vol. 23, no. 6, pp. 1073-1080, June 2012.

[12] S. Jin, D.S. Yeung, and X. Wang, "Network Intrusion Detection in Covariance Feature Space," Pattern Recognition, vol. 40, pp. 21852197, 2007.

[13] C.F. Tsai and C.Y. Lin, "A Triangle Area Based Nearest Neighbors Approach to Intrusion Detection," Pattern Recognition, vol. 43, pp. 222-229, 2010.

[14] A. Jamdagni, Z. Tan, X. He, P. Nanda, and R.P. Liu, "RePIDS: A Multi Tier Real-Time Payload-Based Intrusion Detection System," Computer Networks, vol. 57, pp. 811-824, 2013.

[15] Z. Tan, A. Jamdagni, X. He, P. Nanda, and R.P. Liu, "Denial-ofService Attack Detection Based on Multivariate Correlation Analysis," Proc. Conf. Neural Information Processing, pp. 756-765, 2011.

[16] Z. Tan, A. Jamdagni, X. He, P. Nanda, and R.P. Liu, "TriangleArea-Based Multivariate Correlation Analysis for Effective Denialof-Service Attack Detection," Proc. IEEE 11th Int'l Conf. Trust, Security and Privacy in Computing and Comm., pp. 33-40, 2012.

[17] S.J. Stolfo, W. Fan, W. Lee, A. Prodromidis, and P.K. Chan, "CostBased Modeling for Fraud and Intrusion Detection: Results from the JAM Project," Proc. DARPA Information Survivability Conf. and Exposition (DISCEX'00), vol. 2, pp. 130-144, 2000.

[18] G.V. Moustakides, "Quickest Detection of Abrupt Changes for a Class of Random Processes," IEEE Trans. Information Theory, vol. 44, no. 5, pp. 1965-1968, Sept. 1998.

[19] A.A. Cardenas, J.S. Baras, and V. Ramezani, "Distributed Change Detection for Worms, DDoS and Other Network Attacks," Proc. The Am. Control Conf., vol. 2, pp. 1008-1013, 2004.

[20] W. Wang, X. Zhang, S. Gombault, and S.J. Knapskog, "Attribute Normalization in Network Intrusion Detection," Proc. 10th Int'l Symp. Pervasive Systems, Algorithms, and Networks (ISPAN), pp. 448-453, 2009.

[21] M. Tavallaee, E. Bagheri, L. Wei, and A.A. Ghorbani, "A Detailed Analysis of the KDD Cup 99 Data Set," Proc. IEEE Second Int'l Conf. Computational Intelligence for Security and Defense Applications, pp. 1-6, 2009.

[22] D.E. Knuth, The Art of Computer Programming Vol I: Fundamental Algorithms. Addison-Wesley, 1973.

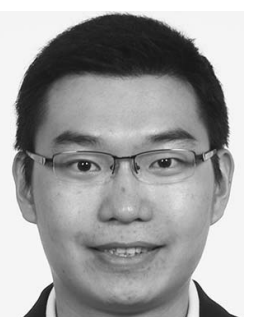

Zhiyuan Tan is working toward the PhD degree at the Faculty of Engineering and Information Technology, University of Technology, Sydney, also a research member of Research Centre for Innovation in IT Services and Applications (iNEXT). His research interests include network security, pattern recognition, machine learning, and P2P overlay network.

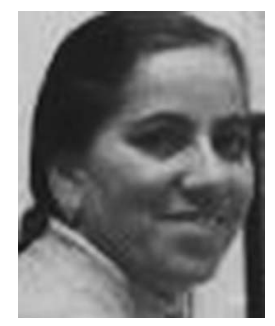

Aruna Jamdagni received the $\mathrm{PhD}$ degree from the University of Technology Sydney, Australia, in 2012. She is a lecturer in the School of Computing and Mathematics, University of Western Sydney, Australia, and a research member of Research Centre for Innovation in IT Services and Applications (iNEXT) at the University of Technology Sydney, Australia. Her research interests include computer and network security and on pattern recognition techniques and fuzzy set theory.

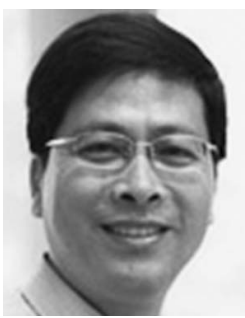

Xiangjian He is a professor of computer science at the School of Computing and Communications. He is also the director of Computer Vision and Recognition Laboratory, the leader of Network Security Research Group, and a deputy director of Research Centre for Innovation in IT Services and Applications (iNEXT), University of Technology, Sydney. His research interests include network security, image processing, pattern recognition and computer vision. $\mathrm{He}$ has been awarded Internationally Registered Technology Specialist by International Technology Institute. He is a senior member of the IEEE. $\mathrm{He}$ is the corresponding author.

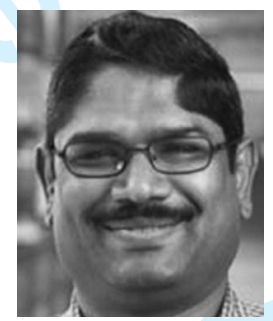

Priyadarsi Nanda is a senior lecturer at the School of Computing and Communications, and is a core research member at the Centre for Innovation in IT Services Applications (iNEXT). His research interests include network QoS, network securities, assisted health care using sensor networks, and wireless networks. He has more than 23 years of experience in teaching and research, and has more than 40 research publications. He is a member of the IEEE.

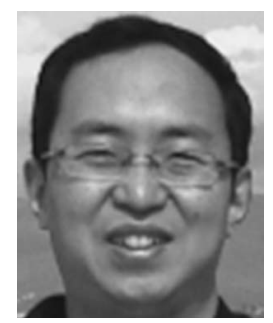

Ren Ping Liu is a principal scientist of networking technology in CSIRO ICT Centre. His research interests include Markov chain modeling, QoS scheduling, and security analysis of communication networks. He has published more than 70 papers in these areas in top journals and conferences. In addition to his research, he has also been heavily involved in and led a number of commercial projects. As a CSIRO consultant, he delivered networking solutions to government and industrial customers, including Optus, AARNet, Nortel, Queensland Health, CityRail, Rio Tinto, and DBCDE. $\mathrm{He}$ is a member of the IEEE.

$\triangle$ For more information on this or any other computing topic, please visit our Digital Library at www.computer.org/publications/dlib. 